ARTICLE

DOI: $10.1038 / s 41467-017-02056-4$

\title{
Bri2 BRICHOS client specificity and chaperone activity are governed by assembly state
}

\author{
Gefei Chen', Axel Abelein', Harriet E. Nilsson (10 2, Axel Leppert', Yuniesky Andrade-Talavera3, \\ Simone Tambaro1, Lovisa Hemmingsson ${ }^{1,4}$, Firoz Roshan ${ }^{3}$, Michael Landreh ${ }^{5,6}$, Henrik Biverstål1,7, \\ Philip J.B. Koeck ${ }^{2}$, Jenny Presto ${ }^{1}$, Hans Hebert (10 ${ }^{2}$, André Fisahn ${ }^{3} \&$ Jan Johansson ${ }^{1}$
}

Protein misfolding and aggregation is increasingly being recognized as a cause of disease. In Alzheimer's disease the amyloid- $\beta$ peptide $(A \beta)$ misfolds into neurotoxic oligomers and assembles into amyloid fibrils. The Bri2 protein associated with Familial British and Danish dementias contains a BRICHOS domain, which reduces $A \beta$ fibrillization as well as neurotoxicity in vitro and in a Drosophila model, but also rescues proteins from irreversible nonfibrillar aggregation. How these different activities are mediated is not known. Here we show that Bri2 BRICHOS monomers potently prevent neuronal network toxicity of $A \beta$, while dimers strongly suppress $A \beta$ fibril formation. The dimers assemble into high-molecular-weight oligomers with an apparent two-fold symmetry, which are efficient inhibitors of non-fibrillar protein aggregation. These results indicate that Bri2 BRICHOS affects qualitatively different aspects of protein misfolding and toxicity via different quaternary structures, suggesting a means to generate molecular chaperone diversity.

\footnotetext{
${ }^{1}$ Department of Neurobiology, Care Sciences and Society, Center for Alzheimer Research, Division of Neurogeriatrics, Karolinska Institutet, 14157 Huddinge, Sweden. ${ }^{2}$ Department of Biosciences and Nutrition, Karolinska Institutet, and School of Technology and Health, KTH Royal institute of Technology, 14183 Huddinge, Sweden. ${ }^{3}$ Department of Neurobiology, Care Sciences and Society, Center for Alzheimer Research, Neuronal Oscillations Lab, Karolinska Institutet, 17177 Stockholm, Sweden. ${ }^{4}$ Department of Physics, Chemistry and Biology, Linköping University, 58183 Linköping, Sweden. ${ }^{5}$ Department of Chemistry, University of Oxford, South Parks Road, Oxford OX15QY, UK. ${ }^{6}$ Science for Life Laboratory, Department of Microbiology, Tumour and Cell Biology, Karolinska Institutet, Tomtebodavägen 23 A, 17165 Stockholm, Sweden. ${ }^{7}$ Department of Physical Organic Chemistry, Latvian Institute of Organic Synthesis, Aizkraukles 21, Riga LV 1006, Latvia. Gefei Chen and Axel Abelein contributed equally to this work. Correspondence and requests for materials should be addressed to J.J. (email: janne.johansson@ki.se)
} 
$\mathrm{M}$ olecular chaperones are essential for cellular homeostasis by promoting the correct folding of proteins over misfolding and aggregation. Under stress conditions, or when the natural protective mechanisms decline, proteins can misfold and self-assemble into large non-fibrillar amorphous aggregates as well as form fibrillar amyloid structures with toxic effects ${ }^{\mathrm{P}-3}$. Non-fibrillar aggregates are associated with human diseases, e.g., cancer and cataract ${ }^{4}$. Self-assembly into amyloid fibrils of specific misfolded proteins is linked to about 40 human diseases, including the severe neurodegenerative disorders Alzheimer's disease (AD) and Parkinson disease, as well as common diseases like type- 2 diabetes ${ }^{5}$. There are many different types and mechanisms of molecular chaperones preventing protein misfolding, ranging from large energy-dependent, multisubunit complexes that are able to sequester and fold entire polypeptide chains in their interior to small chaperones that exert their function by attaching to exposed aggregation-prone regions of (partly) denatured polypeptide chains ${ }^{8-8}$.

The ability to form amyloid fibrils is not limited to the proteins associated with disease, but a myriad of proteins has been shown to form amyloid fibrils in vitro, also generating protein species that are cytotoxic ${ }^{2,9}$. The fact that only $\sim 0.2 \%$ of all available human proteins have been found to form disease-associated amyloid in vivo suggests that efficient defense mechanisms that guard amyloidogenic peptides may exist. Molecular chaperones have been found to prevent amyloid fibril formation in vitro ${ }^{10,11}$ and the heat shock proteins Hsp70 and DNAJB6 were shown to suppress toxic effects of amyloid-forming polyglutamine repeat proteins in transgenic Drosophila melanogaster and mice ${ }^{12}$, 13 .

The most prevalent neurodegenerative disease is $\mathrm{AD}$, for which formation of extracellular amyloid plaques and intracellular neurofibrillary tangles are histopathological hallmarks. $\mathrm{AD}$ development is associated with amyloid fibril formation of the $40-42$ residue amyloid- $\beta$ peptide $(\mathrm{A} \beta)$, in particular the more aggregation-prone $A \beta 42$. Mutations in $A \beta$ precursor protein $(\mathrm{A} \beta \mathrm{PP})$ or its processing enzymes result in early onset $\mathrm{AD}^{14}$. Exactly how dysregulated $A \beta$ homeostasis translates into neurotoxicity and cognitive decline, however, remains to be established. Soluble, oligomeric forms of $A \beta$ are found to be neurotoxic, apparently more than the mature fibrils, and may be key mediators of $\mathrm{AD}$ initiation and progression ${ }^{15}, 16$. A $\beta 42$ fibril formation involves several nucleation steps that display different rate constants; oligomers are formed by primary nucleation from monomers, fibrils are extended by elongation, and oligomers are also formed on the surface of existing fibrils by secondary nucleation reactions ${ }^{17}$. The latter mechanism provides strong positive feedback, which dominates $A \beta 42$ aggregation behavior, and generates the major part of cytotoxic oligomers ${ }^{17}$. Interference with discrete steps in the A $\beta 42$ fibril formation pathways has different effects on the generation of toxic oligomers. Specifically preventing the secondary nucleation markedly reduces the amounts of oligomers, while blocking the fibril elongation event instead increases the amounts of toxic oligomers, although in both cases the overall fibril formation rate is reduced ${ }^{18}$. It is therefore important to delineate the exact mechanisms of fibrillization inhibitors in order to find ways to efficiently reduce $A \beta 42$ neurotoxicity.

The BRICHOS domain (initially found in Bri2, chondromodulin-1 and prosurfactant protein C) is present in a set of proproteins that all have similar overall architecture and harbor regions that are prone to form $\beta$-sheets and to misfold into amyloid. BRICHOS has been proposed to assist the amyloidprone region of their respective proprotein to fold correctly during biosynthesis ${ }^{19,} 20$. Bri2 is produced in several peripheral tissues and in the central nervous system (CNS), with significant expression in neurons of the hippocampus and cerebellum in humans $^{21,22}$. It affects $A \beta P P$ processing ${ }^{23}$ and deposits in $A D$ amyloid plaques $^{24}$. Mutations in Bri2 result in proteolytic release of amyloidogenic peptides (ABri and ADan) and eventually development of familial British or Danish dementias (FBD/FDD), which share clinical and pathological characteristics with $\mathrm{AD}^{22}$, 25. Recombinant human (rh) BRICHOS domains also affect fibril formation of amyloidogenic peptides that are not part of their precursors, including $A \beta 40$ and $A \beta 42^{26,27}$. Transgenic expression of the Bri2 BRICHOS domain alone, i.e., without the proprotein, inhibits A 342 fibril formation and toxicity in Drosophila CNS and eyes $^{28}$. Recently it was shown that a mixture of Bri2 BRICHOS species with different quaternary structures affects the secondary nucleation and fibril elongation steps of $A \beta 42$ fibrillization $^{29}$. In addition, the mixture shows potent general molecular chaperone activity, measured as ability to prevent non-fibrillar aggregation of destabilized model substrate protein ${ }^{28}$. The nature of the secondary nucleation sites is unknown and further studies of BRICHOS effects on A $\beta 42$ fibrillization may provide valuable information on the way in which the secondary pathway functions ${ }^{30}$.

Herein, we find that rh Bri2 BRICHOS can form wellstructured particles with an apparent dihedral symmetry composed of 20-30 subunits, which efficiently inhibit non-fibrillar aggregation of thermo-denatured citrate synthase (CS). a

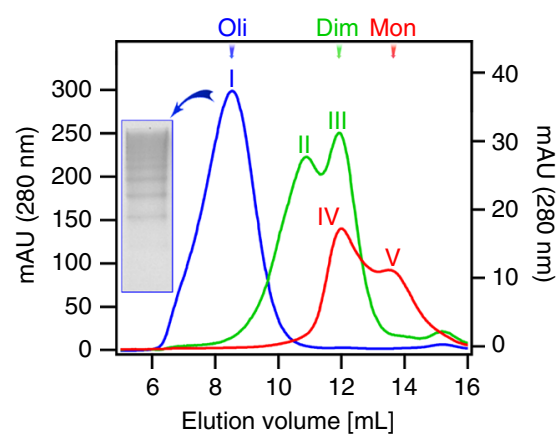

b

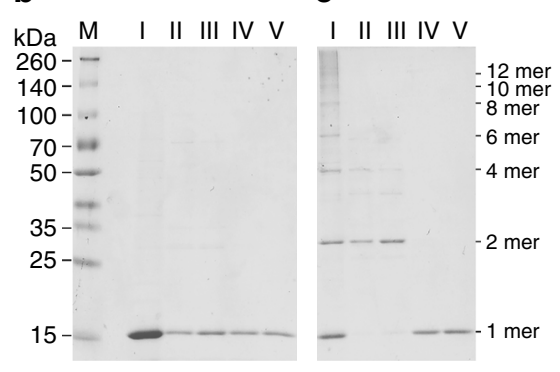

Fig. 1 Quaternary structures of rh Bri2 BRICHOS. a SEC on Superdex 200 PG of rh Bri2 BRICHOS cleaved from individual rh NT*-Bri2 BRICHOS fusion protein species (see Supplementary Fig. 1). Oligomers (blue curve, absorbance scale on the left), dimers (green curve, absorbance scale on the right), and monomers (red curve, absorbance scale on the left). Inset shows native PAGE of the oligomer fraction. The different fractions indicated by Roman numerals in $\mathbf{a}$ were analyzed by SDS-PAGE under reducing $\mathbf{b}$ and non-reducing $\mathbf{c}$ conditions. Lane $\mathrm{M}$ in $\mathbf{b}$ shows migration of protein size markers with masses indicated to the left. The numbers of Bri2 BRICHOS monomer subunits likely present in the different species resolved in $\mathbf{c}$ are given to the right 
a

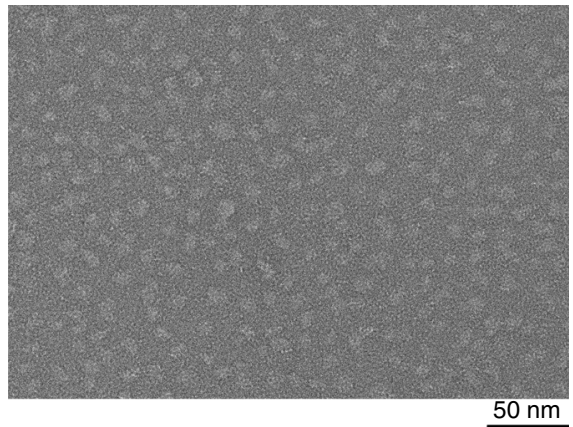

d

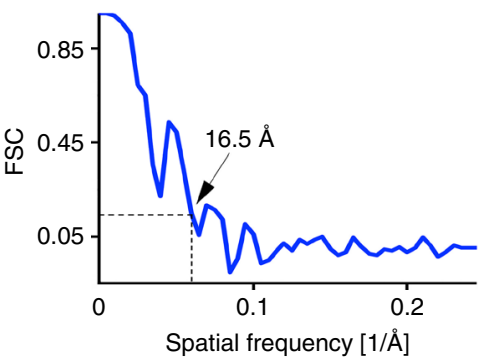

b

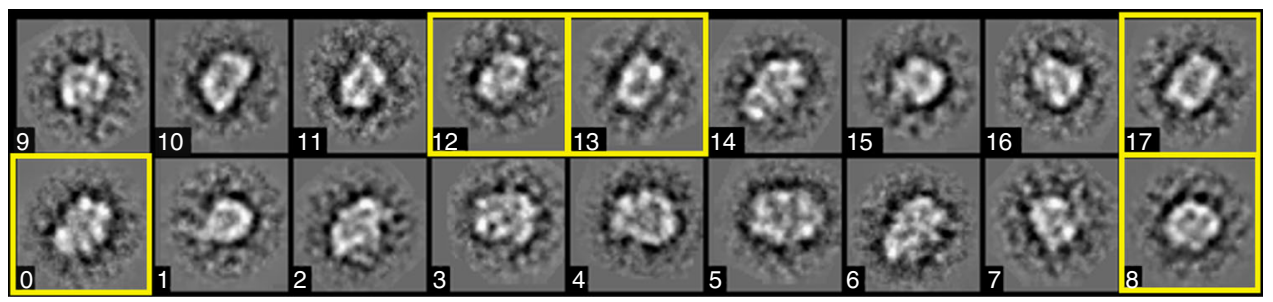

C
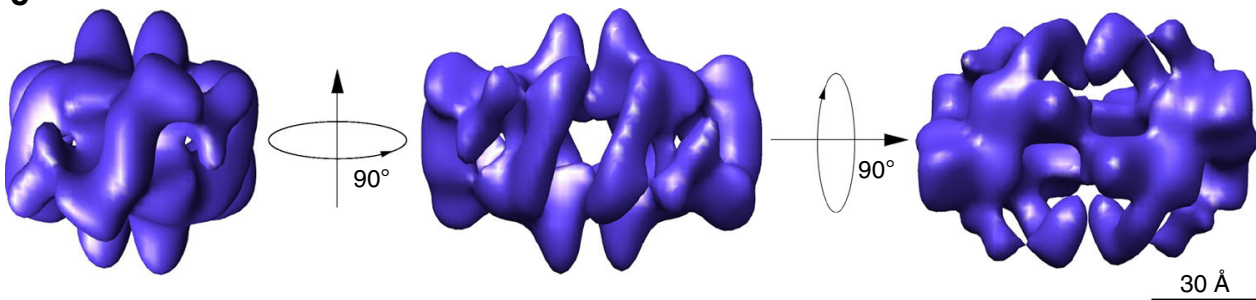

Fig. 2 Electron microscopy analyses of Bri2 BRICHOS oligomers. a Transmission electron micrograph of negatively stained Bri2 BRICHOS oligomers recorded with a JEOL JEM2100F electron microscope and a DE-20 direct electron detector. b Representative 2D-classes of Bri2 oligomers. The class averages are consistent with an approximate 2-fold symmetry. The yellow frames indicate the most obvious 2-fold views. The side of each box side is approximately $22 \mathrm{~nm}$. c 3D density map of Bri2 BRICHOS oligomer with dihedral (D2) symmetry. The viewing directions are parallel to the three different 2 -fold axes. The map was based on 2718 particles extracted from images recorded on a DE-20 detector. The voxel size of the map is $2.076 \AA$. $\mathbf{d}$ The Fourier shell correlation (FSC) curve between reconstructions was produced by splitting the data set in two halves. Both halves were reconstructed separately. The resolution of $\sim 17 \AA$ for the reconstructed 3D density map was calculated from the curve at FSC $=0.143$ (dotted lines)

Monomers and dimers, in contrast, are inactive against nonfibrillar aggregation but efficiently suppress A $\beta 42$ toxicity in hippocampal slice preparations and amyloid fibril formation, respectively.

\section{Results}

Characterization of different rh Bri2 BRICHOS species. To increase yields and allow isolation of distinct quaternary structure species, rh Bri2 BRICHOS was here produced with the new solubility tag $\mathrm{NT}^{\star 31}$. The fusion protein $\mathrm{NT}^{*}$-Bri2 BRICHOS could be resolved into oligomers, dimers and monomers by size exclusion chromatography (SEC) (Supplementary Fig. 1). Isolation of the $\mathrm{NT}^{\star}$-Bri2 BRICHOS oligomers followed by proteolytic release of the BRICHOS domain gave Bri2 BRICHOS oligomers that migrate on SEC as a broad peak centered at an apparent molecular mass of about $370 \mathrm{kDa}$ and show a polydisperse mixture of different sized oligomers on native PAGE (Fig. 1a, blue line, and inset). The oligomers contain an even number of disulfide-linked subunits, at least up to dodecamers, as judged from reducing and non-reducing SDS-PAGE (Fig. 1b, c, lane I). Isolation of $\mathrm{NT}^{\star}$-Bri2 $\mathrm{BRICHOS}$ dimers followed by proteolysis resulted in two fractions upon SEC, with masses corresponding to dimers and tetramers, respectively, and both contain mainly disulfide-dependent dimers (Fig. 1, green line, lanes II and III).
Finally, release of Bri2 BRICHOS from the $\mathrm{NT}^{\star}$-Bri2 BRICHOS fusion protein monomer gave monomers and dimers, both showing exclusively monomers on both reducing as well as non-reducing SDS-PAGE (Fig. 1, red line, lanes IV and V). The protein that eluted under the SEC peak $\mathrm{V}$ is monomeric according to electrospray ionization mass spectrometry (ESI-MS) (Supplementary Fig. 2a) and quantification of free thiols showed that the two cysteines are fully engaged in an intramolecular disulfide (Supplementary Table 1). The ESI-MS of the large oligomers shows a polydisperse appearance that is in line with the differently sized oligomers on native PAGE (Fig. 1a), and dimers were detached by collisional activation (Supplementary Fig. $2 b, c)$, indicating that dimers are constituent building blocks of the oligomers. The monomers studied further herein were narrowly isolated by collecting fraction $\mathrm{V}$ in Fig. 1a, and the dimers and oligomers correspond to fraction III and I, respectively.

Circular dichroism (CD) spectroscopy showed the overall secondary structures are similar for all quaternary structure species, with a somewhat increased content of random coils in the monomer (Supplementary Fig. 2d). ProSP-C BRICHOS, for which there is a crystal structure determined ${ }^{32}$, shows a similar CD spectrum as Bri2 BRICHOS species, albeit with a more pronounced minimum corresponding to random coil structure (Supplementary Fig. 2d), which is compatible with the fact that 
a

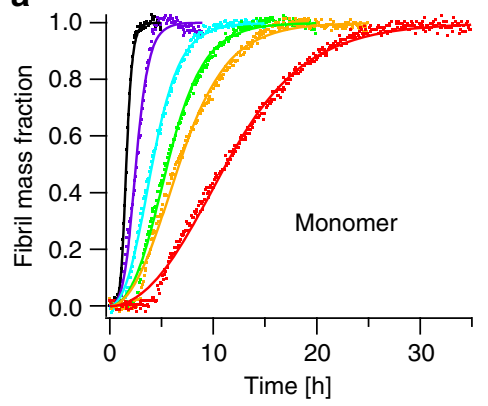

d

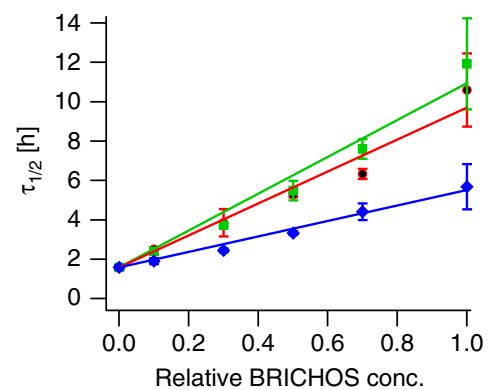

b

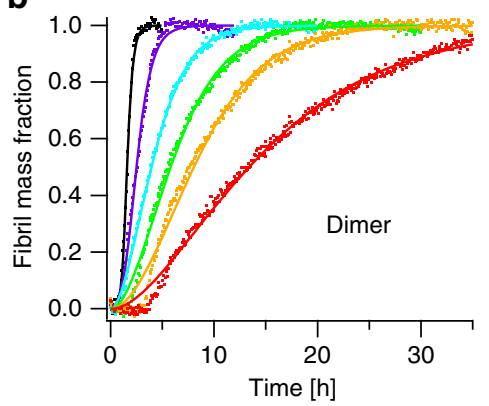

e

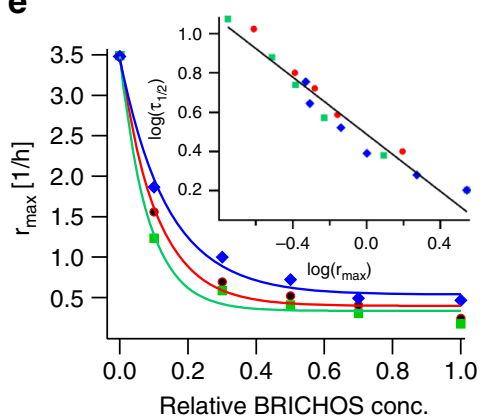

C

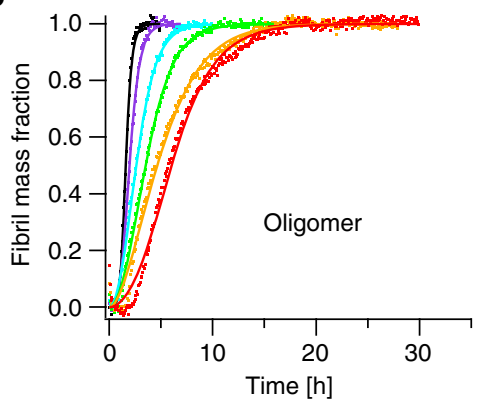

f

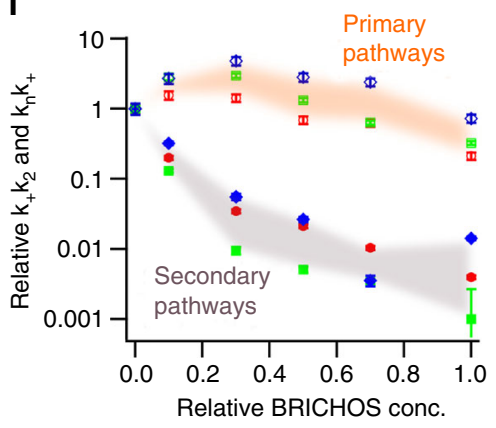

Fig. 3 Kinetics analysis of rh Bri2 BRICHOS species effects on A 42 fibril formation. a-c Individual fits (solid lines) of normalized and averaged aggregation traces (dots) of $3 \mu \mathrm{M} \mathrm{A} \beta 42$ in the presence of 0 (black), 10 (violet), 30 (cyan), 50 (green), 70 (yellow) and 100\% (red) Bri2 BRICHOS (molar percentage referred to monomeric subunits) with the combined rate constants $\sqrt{k_{n} k_{+}}$and $\sqrt{k_{+} k_{2}}$ as free fitting parameters. d, e The sigmoidal fitting parameter $\tau_{1 / 2}$ and $r_{\max }$ exhibit a linear and mono-exponential dependence, respectively, on the relative Bri2 BRICHOS concentration for all species, i.e., the monomer (red), dimer (green) and oligomer (blue). Their logarithmic values fall on a line, indicating the same aggregation mechanism in the presence of all Bri2 BRICHOS species (inset graph). $\mathbf{f}$ The dependencies of the relative combined rate constants obtained from the fits in a-c reveal a strong effect of all Bri2 BRICHOS species on secondary nucleation $\left(k_{+} k_{2}\right.$, closed symbols and gray area), but not on primary $\left(k_{n} k_{+}\right.$, open symbols and orange area) pathways. The data in ( $d-e)$ are presented as means \pm standard deviations of 3-4 replicates of experiments that have been repeated at least five times with qualitatively similar results. The errors in $\mathbf{f}$ are presented as fitting errors

the loop between helices 1 and 2 is longer for proSP-C BRICHOS than for other BRICHOS domains ${ }^{19}$. One typical feature of molecular chaperones is binding to exposed hydrophobic patches of non-native polypeptides ${ }^{33}$. We therefore used the non-polar fluorescent dye bis-ANS for probing the presence of exposed hydrophobic areas of different rh Bri2 BRICHOS quaternary structures. Bis-ANS shows a blue shift of the emission maximum and increased emission intensity upon binding to exposed hydrophobic protein surfaces ${ }^{34}$. When incubated with bis-ANS, all rh Bri2 BRICHOS species gave a marked increase of emission intensity compared with bis-ANS in buffer, and also a blue shift of the emission maximum from about $533 \mathrm{~nm}$ to $480-490 \mathrm{~nm}$ (Supplementary Fig. 2e). Notably, the blue shift is smaller for dimers and monomers compared to the oligomers (Supplementary Fig. 2e). This indicates that all rh Bri2 BRICHOS species have exposed hydrophobic surfaces while the monomer and dimer apparently expose a different hydrophobic environment than the oligomer.

3D reconstruction of rh Bri2 BRICHOS oligomers. To obtain insights into the structural arrangement of rh Bri2 BRICHOS oligomers, we recorded transmission electron microscopy (TEM) micrographs from which $3 \mathrm{D}$ reconstructions can be calculated (Fig. 2a). The micrographs and 2D class averages revealed mostly homogenous assemblies (Fig. 2b). The biochemical analysis (Fig. 1 and Supplementary Fig. 2), showing that dimers $(2 \times 15 \mathrm{kDa})$ assemble into oligomers $(\sim 370 \mathrm{kDa})$, indicates the possibility of a 2 -fold symmetry. Furthermore, the $2 \mathrm{D}$ classification suggests an approximate 2 -fold symmetry, which was thus applied to the $3 \mathrm{D}$ reconstructions. We found that both dihedral
(D2) and cyclic (C2) symmetries gave a stable convergence in the 3D refinement (Supplementary Fig. 3). The refinement with applied C2 symmetry (one symmetry axis) finally converged to resemble the map refined with the higher D2 symmetry (three symmetry axes). Therefore, D2 symmetry was finally selected as shown in Fig. 2c. The resolution obtained in the final maps was $\sim 17 \AA$ with applied D2 symmetry (Fig. 2d), and $18 \AA$ with C2 symmetry (Supplementary Fig. 3) according to the 0.143 FSC criterion. Indicated by the constructed 3D map, the rh Bri2 BRICHOS oligomers are highly structured assemblies, and the clefts and/or tori on the surface are potential hydrophobic binding sites (Fig. 2c), but still a higher resolution 3D map is necessary to elucidate the details. In order to evaluate roughly how many rh Bri2 BRICHOS subunits are able to be held in the constructed 3D map of the large oligomers, we calculated the volumes of the Bri2 BRICHOS monomer model built from proSP-C crystal structure (PDB 2YAD) ${ }^{26}$, and the 3D map of the large oligomers, which are $\sim 9340 \AA^{3}$ and $\sim 221,400 \AA^{3}$ (with threshold set to 1 ), respectively. The volume comparison indicated that around 24 Bri2 BRICHOS subunits could be accommodated in the large oligomers, which is in good agreement with estimated masses of oligomers $(\sim 370 \mathrm{kDa})$ and monomers $(15$ $\mathrm{kDa}$ ) by SEC.

Aß42 fibrillization affected by rh Bri2 BRICHOS species. Shown by the thioflavin T (ThT) fluorescence assay, the rh Bri2 BRICHOS monomer, dimer and oligomer inhibit A $\beta 42$ fibrillization in a dose-dependent manner (Fig. 3), and the aggregation kinetics follows a typical sigmoidal behavior. The appearances of the monomer, dimer and oligomers upon non-reducing SDS- 
a

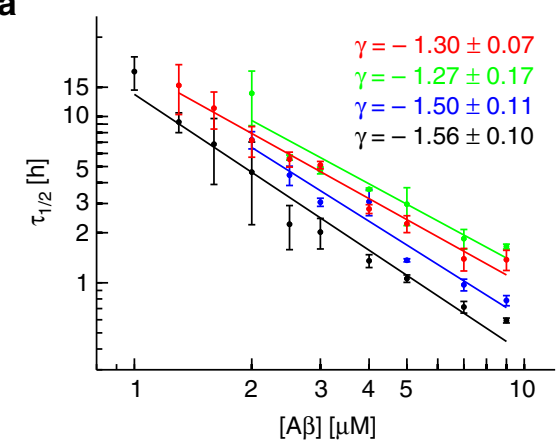

C

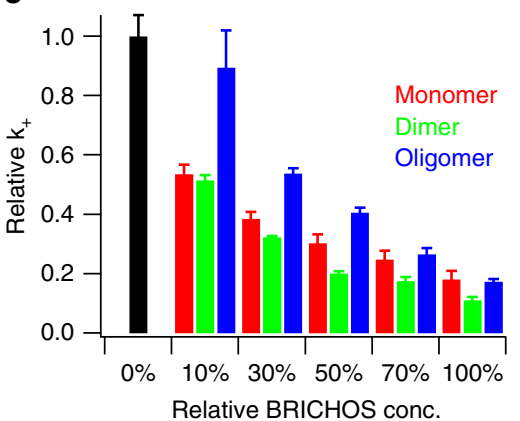

b

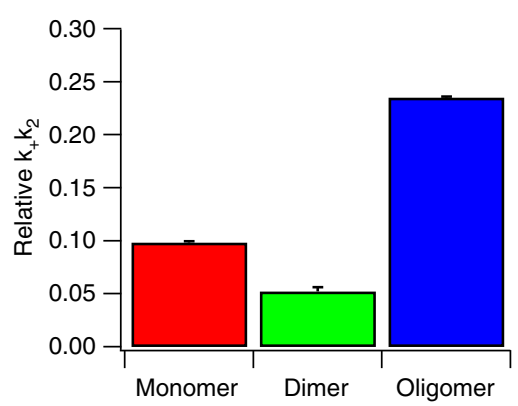

d

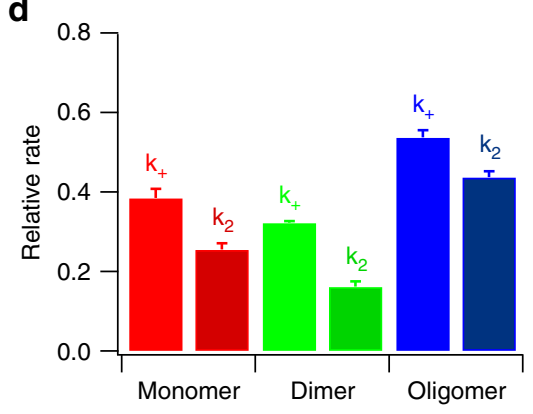

Fig. 4 Effect of rh Bri2 BRICHOS on the nucleation mechanism of A $\beta 42$. a A $\beta 42$ in the absence (black) and presence of $0.9 \mu \mathrm{M}$ Bri2 BRICHOS monomers (red), dimer (green) and oligomer (blue) exhibit a very similar dependence of the aggregation half time, $\tau_{1 / 2}$, on the initial peptide monomer concentration, described by the $\gamma$-exponent. $\mathbf{b}$ A global fit analysis from the data set in a revealed a dominate effect in $k_{+} k_{2}$, related to secondary nucleation and fibril-end elongation, where the Bri2 BRICHOS dimer is the most efficient species. c Estimation of the elongation rates from highly

pre-seeded aggregation kinetics. $\mathbf{d}$ Estimated effect on the individual rate constants $k_{+}$and $k_{2}$ from $\mathbf{b}$ and $\mathbf{c}$ by the different Bri2 $\mathrm{BRICHOS}$ species. The data in $\mathbf{a}$ and $\mathbf{c}$ are presented as means \pm standard deviations of 3-6 replicates of experiments that have been repeated at least five times with qualitatively similar results. The errors of the $\gamma$-exponent in $\mathbf{a}$ and errors presented in $\mathbf{b}$ refer to the fitting errors. The errors in $\mathbf{d}$ are derived from the errors presented in $\mathbf{b}$ and $\mathbf{c}$

PAGE are practically unchanged after $24 \mathrm{~h}$ incubations under the experimental conditions used for the ThT fluorescence assay (Supplementary Fig. 4). The aggregation half time, $\tau_{1 / 2}$, exhibits a linear relation on the Bri2 BRICHOS concentration, while the maximum rate of aggregation, $r_{\max }$, shows a mono-exponential pattern (Fig. 3d, e). At equal mass concentration the rh Bri2 BRICHOS dimer, followed by the monomer, is the most effective structural species that prevents $A \beta 42$ self-assembly. Notably, when correlating the sigmoidal fitting parameters in a doublelogarithmic plot, the values of all rh Bri2 BRICHOS species fall on the same line, indicating that the inhibition mechanism is the same for all quaternary structural arrangements (Fig. 3e, inset).

In general, protein/peptide aggregation kinetics is determined by a set of microscopic nucleation events described by primary $\left(k_{n}\right)$ and secondary $\left(k_{2}\right.$ and $\left.k_{-}\right)$nucleation rate constants in addition to fibril-end elongation $\left(k_{+}\right)^{35}, 36$. Secondary nucleation can be dependent on the protein/peptide monomer concentration, e.g. the formation of a new nucleus on a fibril surface by $n_{2}$ monomers $\left(k_{2}\right)$, or monomer-independent in the case of fibril fragmentation $\left(k_{-}\right)$. The $\gamma$-exponent, defined by $\tau_{1 / 2} \alpha m(0)^{\gamma}$, is determined by the underlying nucleation mechanism ${ }^{35,37}$. We examined the aggregation kinetics at different initial $\mathrm{A} \beta 42$ monomer concentrations, $m(0)$, in the presence of a constant rh Bri2 BRICHOS concentration, revealing roughly identical $\gamma$ exponents for all rh Bri2 BRICHOS species (Fig. 4a and Supplementary Fig. 5), which are in good agreement with previous results for $\mathrm{A} \beta 42$ aggregation alone under quiescent conditions ${ }^{17}$. This hence suggests that also in the presence of Bri2 BRICHOS A $\beta 42$ fibril formation follows mainly monomerdependent secondary pathways.
The set of kinetic equations determined by the microscopic rate constants can be solved analytically as shown by Knowles et al. ${ }^{36-}$ 38 and fitting to the aggregation traces delivers two fitting parameters in form of the combined rate constants $\sqrt{k_{n} k_{+}}$for primary and $\sqrt{k_{+} k_{2}}$ for secondary nucleation, respectively. To quantitatively elucidate the effects of Bri2 BRICHOS on A $\beta 42$ aggregation, we first fitted the kinetic model individually to single aggregation traces of $3 \mu \mathrm{M} \mathrm{A} \beta 42$ in the presence of different Bri2 BRICHOS concentrations (Fig. 3a-c). This analysis revealed that primarily the fitting parameter $\sqrt{k_{+} k_{2}}$ associated to secondary nucleation is affected similarly by all Bri2 BRICHOS species (Fig. 3f). Subsequently, we recorded the aggregation kinetics at different A $\beta 42$ concentration and constant Bri2 BRICHOS concentration. This set of aggregation traces can be fitted globally, where the fitting parameters $\sqrt{k_{n} k_{+}}$and $\sqrt{k_{+} k_{2}}$ are constrained to the same value across all peptide concentrations. We found that the fitting parameter $\sqrt{k_{n} k_{+}}$can be hold to the same value for all Bri2 BRICHOS species as for $A \beta 42$ alone and the fits still describe well the kinetic data set (Supplementary Fig. 5 and Supplementary Table 2), supporting that mainly secondary pathways are modulated by the presence of Bri2 BRICHOS. The results summarized in Fig. $4 \mathrm{~b}$ show that the Bri2 BRICHOS dimer is the most efficient species to inhibit nucleation events related to $k_{+} k_{2}$, i.e., fibril elongation or/and secondary nucleation.

To distinguish which of these nucleation events are predominantly affected, we performed aggregation kinetics in the presence of a high initial seed concentration. Under these conditions aggregation traces typically exhibit a concave aggregation behavior (Supplementary Fig. 6), where the initial slope is 
a

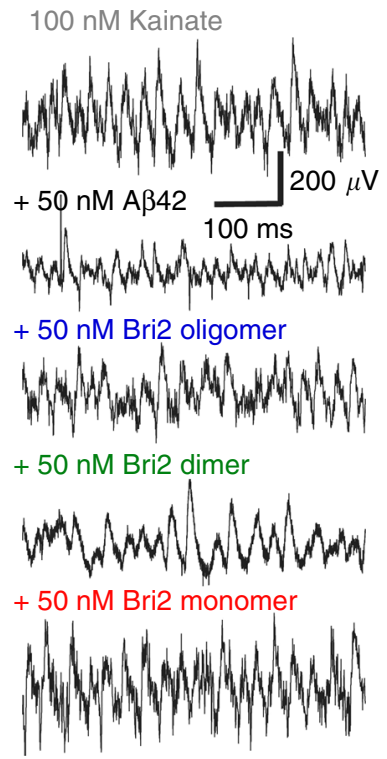

b

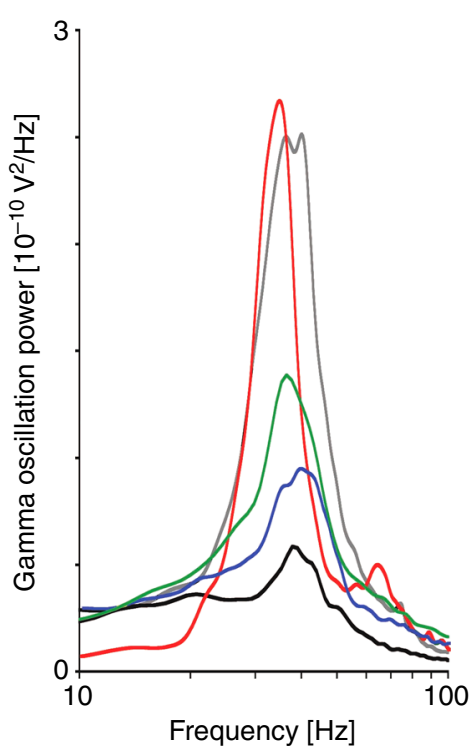

C

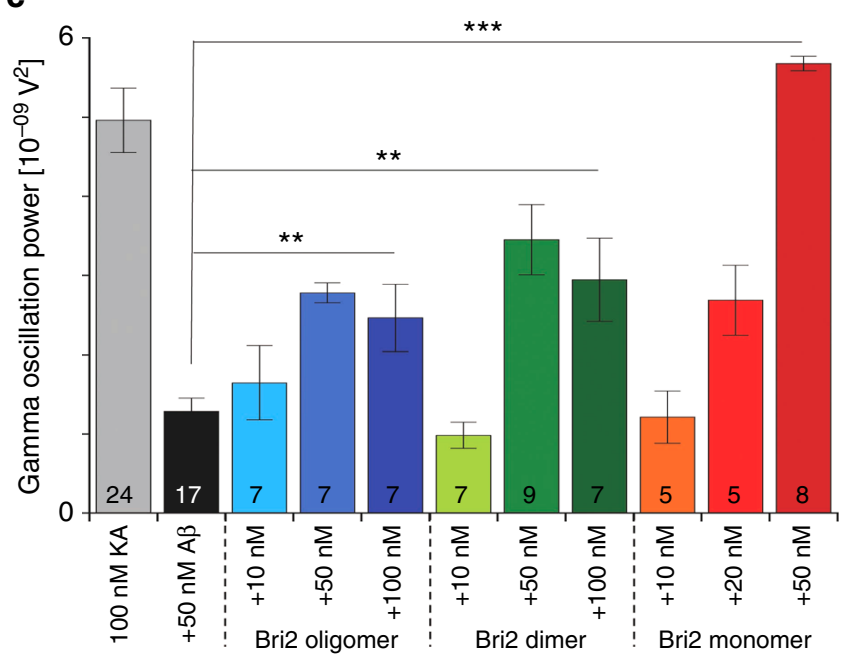

Fig. 5 Effects on A 42 toxicity in mouse hippocampal slices of different $r h$ Bri2 BRICHOS species. a Example traces of $\gamma$ oscillations under control

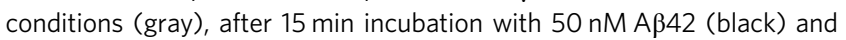

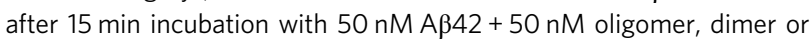
monomer (blue, green and red, respectively). $\mathbf{b}$ Example power spectra of $\gamma$ oscillations under control conditions (gray), after $15 \mathrm{~min}$ incubation with 50 nM A 42 (black) and after 15 min incubation with $50 \mathrm{nM} \mathrm{A \beta 42+50} \mathrm{nM}$ oligomer, dimer or monomer (blue, green and red, respectively). c Summary histogram of $\gamma$ oscillation power under control conditions (gray), after 15 min incubation with $50 \mathrm{nM} \mathrm{A \beta 42} \mathrm{(black)} \mathrm{and} \mathrm{after} 15 \mathrm{~min}$

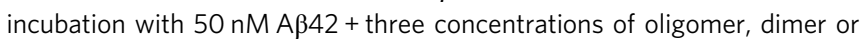
monomer (blue, green and red, respectively). The figures under the histograms denote number of biological replicates, and the data are reported as means \pm standard errors of the means

directly proportional to the elongation rate $k_{+}{ }^{35}$. These experiments revealed that all Bri2 BRICHOS species decrease the elongation rate in a concentration-dependent manner (Fig. 4c and Supplementary Fig. 6). Remarkably, already at low Bri2 BRICHOS concentration, in particular the dimer and to less extent also the monomer, fibril-end elongation is noticeably retarded (Fig. 4c).
To check whether a sole inhibition of the elongation events can describe the observed aggregation behavior, we performed a global fit of the kinetic data set at constant $A \beta 42$ and different Bri2 BRICHOS concentration where the fit was constrained such that only one single rate constant, i.e., $k_{n}, k_{+}$or $k_{2}$, is the sole fitting parameter (Supplementary Fig. 7 and Supplementary Table 3$)^{39}, 40$. As expected from the previous analysis, the primary nucleation rate $k_{n}$ as the sole fitting parameter gave only rise to insufficient fits, underlining that mainly secondary pathways are modulated. However, also a strict constraint where either $k_{+}$or $k_{2}$ is the sole free fitting parameter does not describe the aggregation kinetics adequately. We conclude hence that both fibril-end elongation and secondary nucleation are affected by Bri2 BRICHOS. From the combined rate constants determined from the global fit and the elongation rate from the seeded aggregation kinetics, the rate of secondary nucleation can be estimated at a constant Bri2 BRICHOS concentration (Fig. 4d). This comparison makes evident that the dimer is not only most efficient in suppressing elongation events but also effectively prevents secondary nucleation events catalyzed on the fibril surface.

Visualization of Bri2 BRICHOS interaction with Aß42 fibrils. The kinetic modulation of secondary nucleation and elongation events implicates an association of Bri2 BRICHOS to the fibril surfaces and fibril ends. To visualize this interaction we used antiBri2 BRICHOS immunogold-staining, which enabled us to localize Bri2 BRICHOS on TEM images. These images reveal Bri2 BRICHOS on the A $\beta 42$ fibril surfaces and close to fibril-ends as well (Supplementary Fig. 8d-g), which support the kinetic analysis and provide evidence for an interaction of Bri2 BRICHOS with both the fibril surface and fibril ends. Furthermore, in the presence of rh Bri2 BRICHOS monomer, but not the dimer, A $\beta 42$ small aggregates surrounded by rh Bri2 BRICHOS were observed (Supplementary Fig. 8h, i). This suggests the Bri2 BRICHOS monomers specially bind to non-fibrillar A $\beta 42$ assemblies.

A $\beta 42$ toxicity prevented by different rh Bri2 BRICHOS species. We next tested how the effects on $A \beta 42$ aggregation of the different Bri2 BRICHOS species translated into effects on A $\beta 42$ neurotoxicity. We recorded $\gamma$ oscillations in mouse hippocampal slices after acute exposure to A $\beta 42$ and different rh Bri2 BRICHOS species. In general, the characteristics of $\gamma$ oscillations are important functional biomarkers for brain disorders that involve cognitive decline, since this brain rhythm plays a central role in higher processes, such as learning, memory and cognition ${ }^{41,42}$ Clinical data shows that the cognitive decline observed in $\mathrm{AD}$ patients goes hand-in-hand with a decrease of $\gamma$ oscillations ${ }^{43}$, and it is assumed that the reduction of these network rhythms underlies the negative effects on learning, memory, perception and cognition typical for $\mathrm{AD}$. $\gamma$ oscillations were induced in horizontal hippocampal slices from C57BL/6 mice by superfusing slices with $100 \mathrm{nM}$ kainate (KA) as a control (Fig. 5 and Supplementary Table 4). Pre-incubation of hippocampal slices with $50 \mathrm{nM} \mathrm{A} \beta 42$ for 15 min severely reduced the power of $\gamma$ oscillations generated by subsequent KA application (Fig. 5, KA vs. A $\beta 42: p<0.0001)$.

We found that the individual incubation with all rh Bri2 BRICHOS species reduces A $\beta 42$-induced toxicity (Fig. 5), while control experiments showed that pre-incubation with $50 \mathrm{nM}$ of any rh Bri2 BRICHOS species in the absence of $\mathrm{A} \beta 42$ did not significantly alter the power of $\gamma$ oscillations generated by subsequent KA application (KA vs. rh Bri2 BRICHOS oligomer, $p=0.925$, dimer $p=0.775$, or monomer, $p=0.808$ ). 
a

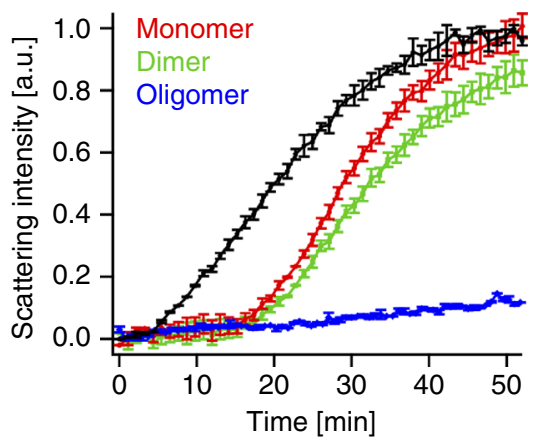

b

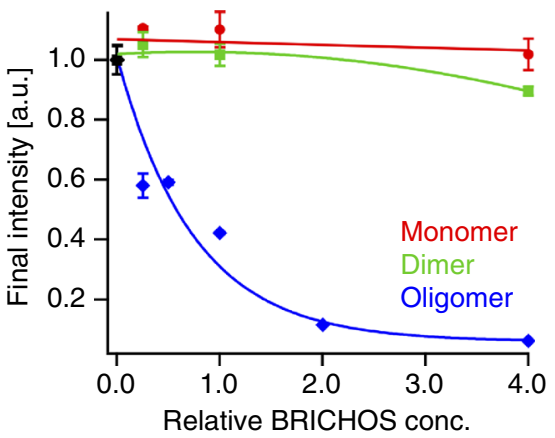

Fig. 6 Ability of different rh Bri2 BRICHOS species to suppress citrate synthase (CS) non-fibrillar aggregation. a Kinetics of aggregation of $600 \mathrm{nM}$ citrate synthase (CS) at $45^{\circ} \mathrm{C}$ alone (black), in the presence of $1.2 \mu \mathrm{M}$ rh Bri2 BRICHOS oligomer (blue), $2.4 \mu \mathrm{M}$ dimer (green) or $2.4 \mu \mathrm{M}$ monomer (red). b Effects of rh Bri2 BRICHOS oligomers (blue), dimers (green) and monomers (red) on CS aggregation at different molar ratios (referred to monomeric subunits) of BRICHOS:CS. The data are presented as means \pm standard deviations of 3-4 replicates of experiments that have been repeated at least five times with qualitatively similar results

a

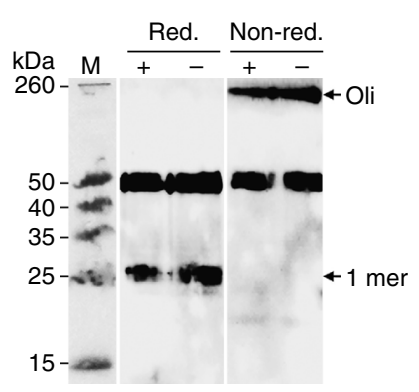

e

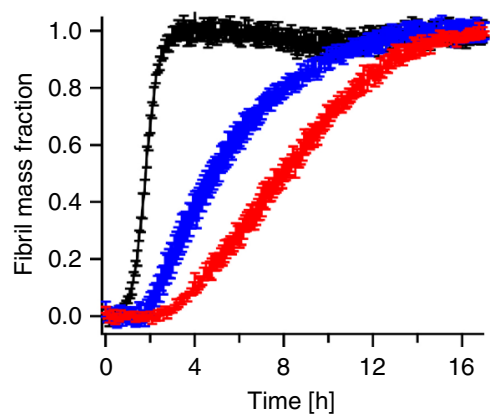

b

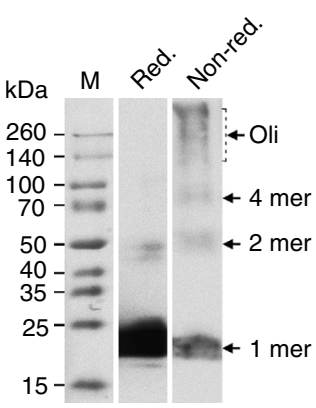

f

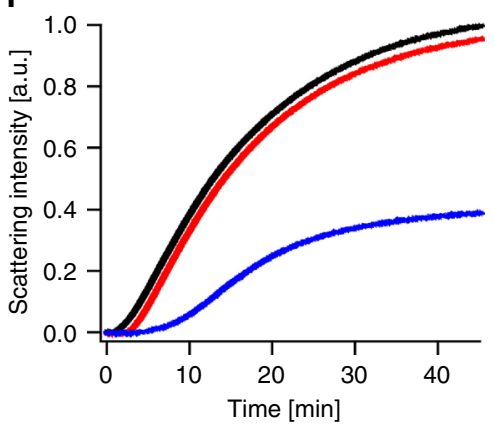

d
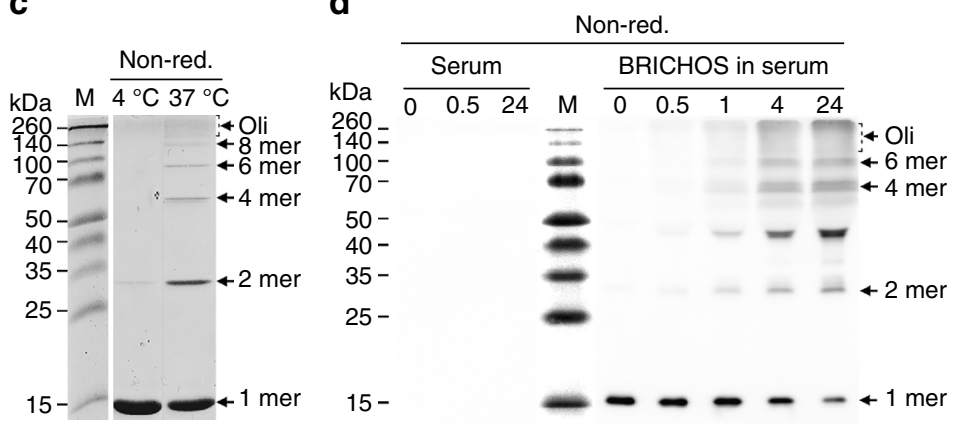

g

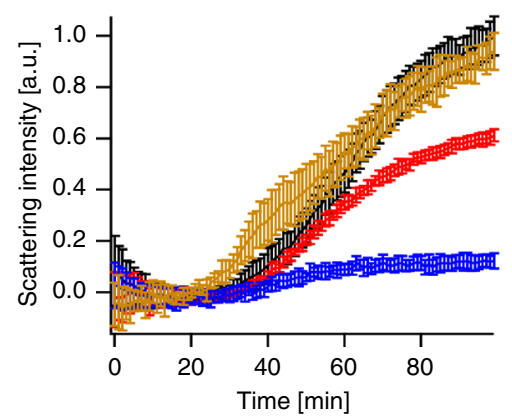

Fig. 7 Quaternary structures and activities of Bri2 BRICHOS secreted from mammalian cells and in serum. a Conditioned culture medium from SH-SY5Y cells was immunoprecipitated for Bri2 BRICHOS and analyzed by SDS-PAGE and Western blot under reducing or non-reducing conditions, and in the presence (+) and absence (-) of $\mathrm{N}$-Ethylmaleimide (NEM). The band at $50 \mathrm{kDa}$ is found after the same treatment of non-conditioned medium. $\mathbf{b}$ Culture medium from HEK293 cells transfected with Bri2 BRICHOS was immunoprecipitated for Bri2 BRICHOS and analyzed by SDS-PAGE and Western blot under reducing or non-reducing conditions. c Monomeric rh Bri2 BRICHOS incubated at $4{ }^{\circ} \mathrm{C}$ or $37^{\circ} \mathrm{C}$ overnight and analyzed by SDS-PAGE under non-reducing conditions. SDS-PAGE of the same samples under reducing conditions is shown in Supplementary Fig. 9c. d Monomeric rh Bri2 BRICHOS incubated in mouse serum at $37^{\circ} \mathrm{C}$, or serum only, analyzed by Western blotting under non-reducing conditions at different time points (in hours) as indicated above each lane. Western blotting of the same samples under reducing conditions is shown in Supplementary Fig. 9 d. e Aggregation kinetics of $3 \mu \mathrm{M}$ A $\beta 42$ alone (black) or with $50 \%$ molar ratio of rh Bri2 BRICHOS monomer incubated at $4^{\circ} \mathrm{C}$ (red) or at $37^{\circ} \mathrm{C}$ overnight (blue). f Aggregation kinetics of $600 \mathrm{nM} \mathrm{CS}$ at $45^{\circ} \mathrm{C}$ alone (black), in the presence of $1.2 \mu \mathrm{M}$ rh Bri2 BRICHOS monomer incubated at $4{ }^{\circ} \mathrm{C}$ (red) or at $37^{\circ} \mathrm{C}$ overnight (blue). $\mathbf{g}$ Aggregation kinetics of $600 \mathrm{nM} \mathrm{CS}$ at $45^{\circ} \mathrm{C}$ in the presence of non-incubated serum (black), serum incubated at $37^{\circ} \mathrm{C}$ (yellow), non-incubated rh Bri2 BRICHOS/serum mixture (red), and rh Bri2 BRICHOS/serum mixture incubated for $24 \mathrm{~h}$ at $37^{\circ} \mathrm{C}$ (blue). The data are presented as means \pm standard deviations of $3-4$ replicates of experiments that have been repeated three times with qualitatively similar results

The rh Bri2 BRICHOS monomer is most efficient in suppressing toxic effects in neuronal network and at a 1:1 molecular ratio it completely prevents $A \beta 42$-induced reduction of $\gamma$ oscillation (Fig. $5 \mathrm{c}$ and Supplementary Table 4, $50 \mathrm{nM} \mathrm{A} \beta 42$ vs. $50 \mathrm{nM} \mathrm{A} \beta 42+50 \mathrm{nM}$ rh Bri2 BRICHOS monomer $p<0.0001)$.
The presence of rh Bri2 BRICHOS dimers and oligomers also reduces the toxic $A \beta 42$ effect on $\gamma$ oscillation power without reaching the level of complete prevention, even for a two-fold excess and the effect levels out after 1:1 A $\beta 42$ : rh Bri2 BRICHOS ratio (Fig. $5 \mathrm{c}$ and Supplementary Table 4 ). 
a

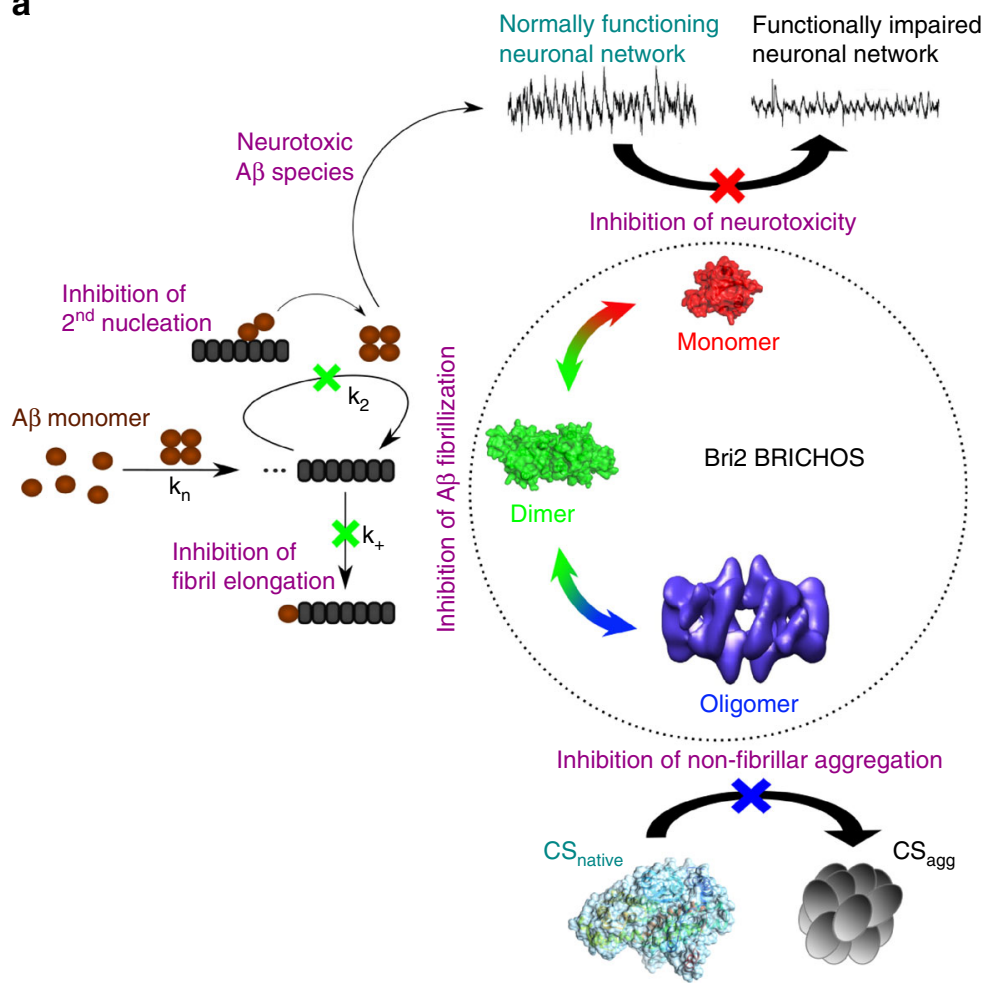

b

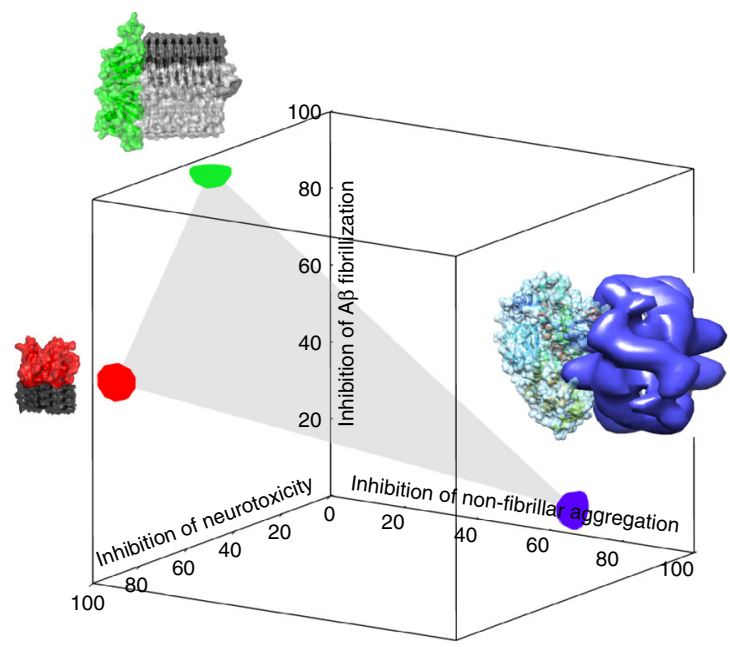

Fig. 8 Different activities of Bri2 BRICHOS are mediated by distinct quaternary structures. a Bri2 BRICHOS monomers form dimers, which are able to assemble into structured high-molecular weight oligomers. Each quaternary structure most efficiently exerts one distinct function: monomers prevent $A \beta 42$-associated neurotoxicity, dimers counteract $A \beta 42$ fibrillization and oligomers inhibit non-fibrillar aggregation. The aggregation behavior of $A \beta$ is governed by different microscopic nucleation events, where in particular fibril end elongation $\left(k_{+}\right)$and surface-catalyzed secondary nucleation $\left(k_{2}\right)$ are inhibited by Bri2 BRICHOS dimers. b The efficiencies in inhibiting neurotoxicity ( $z$-axis), A $\beta 42$ fibrillization ( $y$-axis) and non-fibrillar aggregation ( $x$-axis), derived from data in Figs. 4-6, are plotted for each quaternary structure species. The diversity in functions fits with molecular sizes of the respective Bri2 BRICHOS quaternary structure compared with the sizes of their respective preferred substrate. A Bri2 BRICHOS dimer (surface area $\sim 2570 \AA^{2}$, evaluated by juxtaposing two Bri2 BRICHOS subunit models ${ }^{26}$, built from the proSP-C BRICHOS crystal structure (PDB 2YAD)) matches well the cross-sectional area $\left(\sim 2250 \AA^{2}\right.$ ) of $A \beta 42$ fibrils (PDB 5KK3) that consist of two $\beta$-structured $A \beta$ molecules per fibril layer. A Bri2 BRICHOS monomer, which prevents $A \beta$ toxicity to neuronal networks, fits well in size with single layer $\beta$-structured $A \beta$ molecules that may build up oligomers. A high-molecular weight Bri2 BRICHOS oligomer (Fig. 2) that efficiently suppresses thermo-induced aggregation of CS provides a large binding surface that is suited to capture native and/or partially denatured CS (illustrated by the structure of porcine heart CS, PDB 3ENJ)

Rh Bri2 BRICHOS activities against non-fibrillar aggregation. Unresolved rh Bri2 BRICHOS, i.e., a mixture of different quaternary structures, suppresses aggregation of thermally destabilized citrate synthase (CS) and other substrate proteins, but it has only small effects on refolding capacity ${ }^{28}$. This raises the question whether the ability to suppress non-fibrillar aggregation is specifically associated with any of the now resolved different quaternary structures of $\mathrm{rh}$ Bri2 BRICHOS. By comparing the different species of rh Bri2 BRICHOS, we found that the dimers and monomers are quite inefficient in suppressing thermoinduced aggregation of CS; even at a four-fold molar excess to CS, the reduction in aggregation is far from being substantial (Fig. 6). In sharp contrast, however, the oligomers efficiently reduce aggregation of $600 \mathrm{nM} \mathrm{CS}$ at $45^{\circ} \mathrm{C}$ already at sub-stoichiometric concentrations and completely prevent aggregation at a 2:1 molar ratio to CS (Fig. 6), indicating that the activity against nonfibrillar protein aggregation is specifically associated with Bri2 BRICHOS oligomers.

Bri2 quaternary structures from mammalian cells and in serum. In order to study what quaternary structure species of Bri2 BRICHOS that are found in mammalian cells we used SHSY5Y and HEK293 cells. Immunoprecipitation and Western blot of conditioned medium from non-transfected SH-SY5Y cells using an anti Bri2 BRICHOS antibody, revealed Bri2 BRICHOS monomer around $25 \mathrm{kDa}$ under reducing conditions, and high molecular weight oligomers under non-reducing conditions, also when the thiol-blocking agent $N$-ethylmaleimide (NEM) is present during sample preparation (Fig. 7a). This suggests that the BRICHOS domain that is cleaved out from endogenous Bri2 expressed and secreted from SH-SY5Y cells, mainly forms larger disulfide-linked oligomers. We also recombinantly expressed Bri2 BRICHOS domain directed to the secretory pathway in HEK293 cells, and monomers, dimers, tetramers and oligomers were detected in the medium (Fig. 7b) and in cell lysates (Supplementary Fig. 9a). PNGase treatment confirms that rh Bri2 BRICHOS expressed on its own, i.e., without the rest of the Bri2 protein, gets glycosylated (Supplementary Fig. 9b), like the fulllength protein ${ }^{44}$, indicating that glycosylation of the BRICHOS domain does not affect formation of quaternary structure species.

In order to gain insights into the oligomerization mechanism of rh Bri2 BRICHOS, monomeric species from $E$. coli were isolated and incubated under different conditions. The monomeric rh Bri2 BRICHOS converts to disulfide-dependent dimers and even-numbered oligomers when incubated at $37^{\circ} \mathrm{C}$ over night in a phosphate buffer $\mathrm{pH} 8.0$, but not at $4^{\circ} \mathrm{C}$ (Fig. $7 \mathrm{c}$ and Supplementary Fig. 9c). We also tried to mimic a physiological 
environment, and incubated monomeric rh Bri2 BRICHOS in the presence of mouse serum at $37^{\circ} \mathrm{C}$, which similarly resulted in formation of disulfide-dependent dimers, tetramers, hexamers and oligomers within $0.5-1 \mathrm{~h}$ (Fig. 7d and Supplementary Fig. 9d). This indicates that Bri2 BRICHOS monomers can form dimers, which generate Bri2 BRICHOS oligomers with even number of subunits, and that different quaternary structure species coexist under physiological conditions. The relative occurrence of oligomers may be underestimated in these experiments since SDS-PAGE allows detection only of covalently linked oligomers.

Finally, we determined how conversion from rh Bri2 BRICHOS monomers to dimers and oligomers in buffer or serum affect the abilities to reduce CS aggregation and A $\beta 42$ fibril formation, respectively. The rh Bri2 BRICHOS monomers, present after incubation in phosphate buffer at $4{ }^{\circ} \mathrm{C}$, delayed A $\beta 42$ fibrillization in the same manner as non-incubated monomers. In contrast, incubation at $37^{\circ} \mathrm{C}$ overnight, which result in formation of dimers and oligomers (Fig. 7c), gives decreased anti A $\beta 42$ fibrillization ability (Fig. 7e). The effects on the ability to inhibit CS aggregation, however, are just the opposite; after incubation at $37^{\circ} \mathrm{C}$ the potency to prevent $\mathrm{CS}$ aggregation is increased (Fig. 7f). Likewise, the incubation of rh Bri2 BRICHOS monomers in serum, which also results in formation of oligomers (Fig. 7d), gives increased ability to prevent CS aggregation (Fig. 7g). As a control, we incubated the trimeric proSP-C BRICHOS ${ }^{28,} 32$ at $37^{\circ} \mathrm{C}$ overnight and found neither detectable formation of disulfide linked oligomers nor effects on the ability to reduce $\mathrm{A} \beta 42$ fibril formation (Supplementary Fig. 9e, f).

\section{Discussion}

In the present work, we show that one single protein, the Bri2 BRICHOS domain, possesses three distinct activities: it inhibits A $\beta 42$ fibril formation, $A \beta$-induced neurotoxicity and non-fibrillar protein aggregation, and these activities are associated with different quaternary structure species. The Bri2 BRICHOS monomers are able to spontaneously convert into dimers and subsequently assemble into high-molecular-weight oligomers with dimeric subunits. Notably, this quaternary structure diversity is found for recombinant Bri2 BRICHOS from both prokaryotic and eukaryotic hosts as well as for endogenous Bri2 BRICHOS secreted from SH-SY5Y cells. Moreover, isolated monomeric rh Bri2 BRICHOS converts into different quaternary structures after incubation under physiological-like conditions, which is accompanied with altered chaperone function.

The importance of protein multimerization has been recognized in relation $A \beta$ generation, in the context of $\gamma$-secretase mediated cleavage of $\mathrm{A} \beta \mathrm{PP}^{45}$. Our results suggest that multimerization of Bri2 BRICHOS regulates its chaperone activity and thereby its ability to affect $A \beta$ fibrillization and neurotoxicity. Recombinant Bri2 BRICHOS high-molecular-weight oligomers efficiently inhibit non-fibrillar aggregation of thermo-denatured CS while both monomers and dimers are basically inactive and do not show any enhanced inhibitory effects (Fig. 6). In contrast, Bri2 BRICHOS dimers most efficiently suppress A $\beta 42$ fibrillization, in particular elongation and secondary nucleation events. Also the monomeric, and to a lesser extent the oligomeric, forms retard A $\beta 42$ self-assembly, while the dimeric structure appears to be most suited to prevent surface-catalyzed nucleation and fibrilend elongation (Figs. 3 and 4). It is crucial to find potential AD drug candidates not only by their ability to non-specifically suppress amyloid formation, but rather by their efficiency in suppressing high levels of neurotoxic oligomers. The Bri2 BRICHOS monomers are most efficacious in reducing $A \beta 42$-induced toxicity in neuronal networks and completely prevent toxic effects at a 1:1 A $\beta 42: B R I C H O S$ molar ratio. The dimer and oligomer can also prevent $A \beta 42$-induced damage to the neuronal network, yet their effects are limited and do not reach $100 \%$ prevention even at a higher 1:2 A 342 :BRICHOS molar ratio. The lack of correlation between potency in inhibiting A $\beta 42$ fibril formation and neurotoxicity supports the concept that assemblies formed during fibril formation, rather than the fibrils as such, are the main culprits in mediating toxicity.

We rationalized these results in a schematic model (Fig. 8). It is striking that the same chaperone domain can execute conceptually different functions, which are essential for the successful operation of the cell under proteostasis stress. Different quaternary structures are not only adopted by the Bri2 BRICHOS domain, but also other molecular chaperons can assemble into differently sized species, yet in a different manner from Bri2 BRICHOS. ProSP-C BRICHOS, phylogenetically in the same family as the Bri2 BRICHOS, is uniquely expressed in the lung epithelium, and the recombinant proSP-C BRICHOS mainly forms trimers. The trimer appears to be an inactive storage form, while the monomer is the active species against amyloid fibrillization $^{32,46}$. Interestingly, neither as trimer nor monomer, proSP$\mathrm{C}$ BRICHOS possesses general chaperone activity and is not able to inhibit non-fibrillar aggregation ${ }^{28}$. This is in stark contrast to Bri2 BRICHOS, suggesting that BRICHOS domains from different proproteins have adopted diverse quaternary structures and functions during evolution.

Small heat-shock proteins (sHSP) are a large molecular chaperone family referred to as holdase chaperones and an important common feature of most sHSPs is the ability to assemble into large oligomers. These oligomeric assemblies are built up by dimeric blocks, which typically range from 12 to $>32$ subunits. The differently sized assemblies are mediated by non-conserved $\mathrm{N}$ - and C-terminal segments ${ }^{47-49}$. For performing efficient chaperone activity, most sHSPs require disassembling from inactive oligomers to smaller active species ${ }^{50}$. Also, a bias toward monomeric substructure of $\alpha$-crystallins was proposed to be responsible for the aberrant chaperone behavior associated with protein deposition disease ${ }^{51}$. This mechanism is in sharp contrast to Bri2 BRICHOS, where large oligomers are the active species preventing non-fibrillar aggregation (Fig. 8).

Some molecular chaperones, including sHSP, also show inhibitory effects against $\mathrm{A} \beta$ fibrillization in vitro, e.g., Hsp40, Hsp70, Hsp90, Hsp104, $\alpha \mathrm{A}$-, and $\alpha \mathrm{B}$-crystallin ${ }^{29}, 52-54$. The diverse antiamyloid active chaperones affect different microscopic nucleation events in A $\beta$ fibril formation. DNAJB6 from the Hsp40 family inhibits predominately primary nucleation ${ }^{29}$, proSP-C BRICHOS prevents secondary nucleation ${ }^{18}$ and $\alpha \mathrm{B}$-crystallin modulates both elongation and secondary nucleation events ${ }^{29}$, similarly as the comprehensive abilities of Bri2 BRICHOS, indicating that molecular chaperones have evolved specific capacities to interact with different $A \beta$ species $^{29}$. $\alpha B$-crystallin was recently reported to use different interfaces to capture amorphous and amyloid clients, respectively. A hydrophobic edge of the central $\beta$-sandwich of $\alpha \mathrm{B}$-crystallin preferentially binds $\mathrm{A} \beta$ peptides, while amorphously aggregating clients are captured by the partially disordered N-terminal domain ${ }^{55}$. Inhibitory functions that are associated with distinct binding sites for amorphous and amyloid aggregates, as for $\alpha \mathrm{B}$-crystallin, are conceptually different to a quaternary structure dependent functional diversity, which is exemplified by Bri2 BRICHOS. In addition to sHSPs, a number of other molecular chaperones or chaperone-like proteins have been reported to inhibit amyloid formation, among them clusterin and calcium-binding protein nuclebindin- $1^{56}, 57$. Clusterin can assemble into a variety of oligomeric forms built up by heterodimers via non-covalent association ${ }^{56}$. Clusterin was reported to suppress non-fibrillar aggregation induced by stress and to inhibit 
$A \beta$ fibrillization as well as to reduce $A \beta$-associated toxicity, and the dissociation into dimers enhances its chaperone activity ${ }^{56,58,}$ 59 . Even though many aspects of the mechanism of action are still unclear, it appears that the functioning of clusterin's protective chaperone activities is similar to sHSPs, but separated from Bri2 BRICHOS.

In contrast to many other molecular chaperones, the oligomeric form of Bri2 BRICHOS most efficiently acts against nonfibrillar aggregation, which might be related to distinct structural arrangement and binding surface. Indeed, a change of the exposed hydrophobic surface upon oligomer formation is indicated by a modulated bis-ANS fluorescence (Supplementary Fig. 2e). Hence, larger and/or more exposed hydrophobic regions may be present in the oligomeric assemblies for substrate binding. Furthermore, the distinct symmetric structure of Bri2 BRICHOS oligomers presented in this study provides the capability to efficiently capture native and/or partly denatured CS (Fig. 8b).

The dimeric structure of Bri2 BRICHOS is superior in inhibition of A $\beta 42$ fibril formation compared to the Bri2 BRICHOS monomer and oligomer. We found that both elongation and secondary nucleation events are inhibited most efficiently in the presence of the Bri2 BRICHOS dimer, suggesting that the dimer more comprehensively covers the fibril-ends and the fibril surface. Compared to the Bri2 BRICHOS monomers, the absolute number of dimers is only half at the same mass concentration, indicating that the dimer binds much stronger to the A $\beta 42$ fibrilend and shields the fibril surface more efficiently from secondary nucleation. For the Bri2 BRICHOS oligomer, crucial amyloid interacting surfaces might be, at least partly, buried in the interior of the structure, thereby reducing its inhibitory effect against A $\beta 42$ fibril formation. Moreover, the Bri2 BRICHOS oligomer is present in a much smaller absolute number than the dimer, diminishing the ability to effectively cover fibril surfaces and ends. Recently, two independent studies reported a 3D A $\beta 42$ fibril structure with two $A \beta 42$ molecules per fibril layer, forming a dimeric cross- $\beta$ sheet entity ${ }^{60,61}$. The size of the Bri2 BRICHOS dimer matches remarkably well the cross-sectional area of such a dimeric $\beta$-sheet motif, facilitating a large interaction surface and efficient binding (Fig. 8b).

The monomeric form of Bri2 BRICHOS most effectively suppresses $A \beta 42$-induced neurotoxicity, reaching a prevention effect of $\gamma$-oscillation up to $100 \%$, whereas the other Bri2 BRICHOS species do not yield complete prevention. Considering the present results, it appears that the small qualitative differences among the different Bri2 BRICHOS species in the $k_{2} / k_{+}$ratio, which is related to the amount of generated A $\beta 42$ oligomers, are dependent on the present Bri2 BRICHOS concentration (Figs. 3 and 4). This makes extrapolation of the Bri2 BRICHOS effects on A $\beta 42$ fibrillization to neurotoxicity experiments on hippocampal slices difficult, as they were performed in a very different concentration range. It is however striking that the size of the Bri2 BRICHOS monomer well fits a single $\beta$-sheet surface (Fig. 8b). A $\beta$ oligomers, in contrast to mature fibrils ${ }^{60,61}$, can feature such a monomeric $\beta$-sheet interface, providing a binding surface for monomeric Bri2 BRICHOS. Such an interaction is also indicated by immunogoldTEM staining images (Supplementary Fig. 8d-i), revealing accumulation of Bri2 BRICHOS monomers, but not dimers, around small non-fibrillar objects, putative pre-fibrillar $A \beta$ species. Preferential binding of the Bri2 BRICHOS monomer to small sized $\mathrm{A} \beta$ species may thus be the origin for an enhanced protective effect against neurotoxicity.

Bri2 undergoes several proteolytic cleavages; furin-like proteinase generates a $\mathrm{C}$-terminal peptide and a membrane bound mature Bri2 that is further processed by ADAM10, which sheds the BRICHOS domain into the extracellular space ${ }^{62}$. Recombinant Bri2 proteins expressed in HEK293 cells form dimers and oligomers via non-covalent interactions and/or disulfide bonds formed in the ER, and disulfide linked dimers were also detected in mouse brains ${ }^{63}$. Recently, Bri2 levels were found to increase up to 3 -fold and to form more high molecular weight oligomers in $\mathrm{AD}$ brain compared to healthy controls ${ }^{24}$. This suggests that the spectrum of Bri2 BRICHOS quaternary structures and activities (Fig. 8) can be affected by physiological as well as pathological processes. Further studies in vivo are required to test these suppositions.

In conclusion, Bri2 BRICHOS has the ability to execute distinct chaperone-like activities, i.e., prevention of non-fibrillar and fibrillar aggregation as well as a neuroprotective function, via its different assembly states. Distinct chaperone-like functions from one chaperone domain provides a means to generate molecular chaperone diversity, which potentially can be implemented in treatment strategies against amyloid-related and other proteinmisfolding diseases.

\section{Methods}

Rh Bri2 BRICHOS preparation. A gene fragment encoding $\mathrm{NT}^{\star}$-Bri2 BRICHOS (corresponding to residue 113-231 of full-length human Bri2) was cloned into a modified pET vector (primer sequences are given in Supplementary Table 5). NT is an efficient solubility tag for recombinant protein production derived from the $\mathrm{N}$-terminal domain of spider silk proteins ${ }^{31}$. The construct coding for the fusion protein $\mathrm{His}_{6}-\mathrm{NT}^{*}$-thrombin cleavage site-Bri2 BRICHOS was transfected into SHuffle T7 competent E. coli (K12 strain) cells. The cells were incubated at $30^{\circ} \mathrm{C}$ in $\mathrm{LB}$ medium containing $15 \mu \mathrm{g} \mathrm{mL}^{-1}$ kanamycin, at an $\mathrm{OD}_{600 \mathrm{~nm}}$ around 0.9 , the incubation temperature was lowered to $20^{\circ} \mathrm{C}$, and Isopropyl $\beta$-D-1-

thiogalactopyranoside (IPTG) was added to $0.5 \mathrm{mM}$ and the cells were incubated overnight. The cells were then harvested by $7,000 \times \mathrm{g}$ centrifugation at $4{ }^{\circ} \mathrm{C}$, after

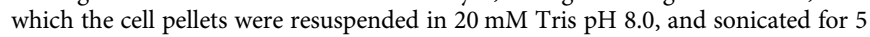
min on ice ( $2 \mathrm{~s}$ on, $2 \mathrm{~s}$ off, $65 \%$ of max power). The lysate was centrifuged $(50,000 \times \mathrm{g})$ at $4^{\circ} \mathrm{C}$ for $30 \mathrm{~min}$ and the supernatant containing the fusion protein was then loaded on a Ni-NTA column. Unbound proteins were washed away and the fusion protein was eluted with $300 \mathrm{mM}$ imidazole, and then dialyzed overnight against $20 \mathrm{mM}$ Tris $\mathrm{pH} 8.0$ at $4{ }^{\circ} \mathrm{C}$. To remove the $\mathrm{His}_{6}-\mathrm{NT}^{*}$ part, the fusion proteins were cleaved with thrombin (1:1000 enzyme to substrate, w/w) at $4{ }^{\circ} \mathrm{C}$ overnight and loaded over a Ni-NTA column.

Rh Bri2 BRICHOS species isolated by SEC. Different rh Bri2 BRICHOS species were separated and analyzed by Superdex 200 PG, 200 GL or 75 PG columns (GE Healthcare, UK) using an ÄKTA basic 10 FPLC system (GE Healthcare, UK). Molecular mass standards aprotinin $(6.5 \mathrm{kDa})$, ribonuclease $(13.7 \mathrm{kDa})$, carbonic anhydrase $(29 \mathrm{kDa})$, ovalbumin $(44 \mathrm{kDa})$, conalbumin $(75 \mathrm{kDa})$, aldolase $(158 \mathrm{kDa})$ and ferritin $(440 \mathrm{kDa})$ (GE Healthcare, UK) were used for calibration. For preparation of rh Bri2 BRICHOS oligomers for EM density map determination, a Superose 6 GL column (GE Healthcare, UK) was used.

ESI-MS on rh Bri2 BRICHOS species. Prior to ESI-MS analysis, rh Bri2 BRICHOS fractions upon SEC were exchanged into $200 \mathrm{mM}$ ammonium acetate $\mathrm{pH} 7.5$ using BioSpin microcentrifuge columns (BioRad, US). Final protein concentrations (referred to monomeric subunit) were $\sim 80 \mu \mathrm{M}$ for rh Bri2 BRICHOS oligomers and $20 \mu \mathrm{M}$ for the monomers. Spectra were recorded on a Waters Synapt G1 mass spectrometer (Waters, Milford, MA) modified for high mass analysis. Samples were introduced into the mass spectrometer using in-house produced gold-coated borosilicate capillaries. Instrument settings were: Capillary voltage $1.5 \mathrm{~V}$, sample cone voltage $30 \mathrm{~V}$, extraction cone voltage $4 \mathrm{~V}$. The collision voltages in the trap were step-wise increased from $10 \mathrm{~V}$ to $180 \mathrm{~V}$ in $10 \mathrm{~V}$ increments. The transfer voltage was $10 \mathrm{~V}$. The source pressure was increased to 7 mbar. Trap gas was $\mathrm{N}_{2}$ with a flow rate of $8 \mathrm{~mL} \mathrm{~h}^{-1}$. Data analysis was performed using Waters MassLynx 4.1 software.

Quantification of disulfide bonds. Rh Bri2 BRICHOS monomers were prepared in native (100 mM Tris $\mathrm{pH} 8.0$ ), denaturing (6 M guanidine hydrochloride (Gdn$\mathrm{HCl}$ ) in $100 \mathrm{mM}$ Tris $\mathrm{pH} 8.0$ ) or reducing buffer (denaturing buffer with 20 fold molar excess of tris(2-carboxyethyl)phosphine (TCEP) over protein concentration). Excess of TCEP was removed after $16 \mathrm{~h}$ reduction at room temperature by two rounds of buffer exchange in degassed denaturing buffer over a PD-10 desalting column (GE Healthcare, UK). Samples were prepared in $1 \mathrm{~mL}$ acryl cuvettes, containing $1 \mathrm{~mL}$ one of above mentioned buffer solutions, $300 \mathrm{nmol}$ DTNB (Sigma-Aldrich, Germany) and 2-4 nM Bri2 BRICHOS proteins. Free thiols were quantified by monitoring the absorbance at $412 \mathrm{~nm}$ (UV-1800, Shimadzu). The amount of released TNB2- was calculated using a molar absorption coefficient of $14,150 \mathrm{M}^{-1} \mathrm{~cm}^{-1}$ in Tris buffer and $13,700 \mathrm{M}^{-1} \mathrm{~cm}^{-1}$ in Gdn-HCl. 
CD spectroscopy and bis-ANS fluorescence. CD spectra were recorded from 260 to $190 \mathrm{~nm}$ at $25^{\circ} \mathrm{C}$ in $1 \mathrm{~mm}$ path length quartz cuvettes using an Aviv $410 \mathrm{Spec}$ trometer (Lakewood, NJ, USA). The wavelength step was $0.5 \mathrm{~nm}$, averaging time $0.3 \mathrm{~s}$, time constant $100 \mathrm{~ms}$, and bandwidth $1 \mathrm{~nm}$. The spectra shown are averages of three consecutive scans. Fluorescence experiments were performed with $1 \mu \mathrm{M}$ rh Bri2 BRICHOS oligomer, dimer or monomer in $5 \mathrm{mM}$ Tris $\mathrm{pH} 7.4$, incubated with $2 \mu \mathrm{M}$ bis-ANS for $10 \mathrm{~min}$ at $25^{\circ} \mathrm{C}$. Fluorescence measurements were performed with a SLM-Aminco AB-2 spectrofluorimeter by using thermostated cuvette holder (Thermo Spectronic, Waltham, MA, USA). The fluorescence emission spectra of bis-ANS from 420 to $600 \mathrm{~nm}$ were recorded after excitation at $395 \mathrm{~nm}$.

Preparation and TEM imaging of Bri2 BRICHOS oligomers. Rh Bri2 BRICHOS oligomers were isolated in $20 \mathrm{mM}$ ammonium acetate $\mathrm{pH} 8.0$ with a Superose $6 \mathrm{GL}$ column, and a narrow peak fraction with protein concentration of $47 \mu \mathrm{g} \mathrm{mL}^{-1}$ was collected. Immediately, the collected fraction was stored on ice followed by grid preparation. Aliquots $(4 \mu \mathrm{L})$ were adsorbed onto glow-discharged continuous carbon-coated copper grids (400 mesh, Analytical Standards) for $2 \mathrm{~min}$. The grids were subsequently blotted with filter paper, washed with two drops of milli-Q water and negatively stained with one drop of $2 \%(\mathrm{w} / \mathrm{v})$ uranyl acetate for $45 \mathrm{~s}$ before final blotting and air-drying. The sample was imaged using a Jeol JEM2100F field emission gun transmission electron microscope (Jeol, Japan) operating at $200 \mathrm{kV}$. Single micrographs for evaluating quality of the sample were recorded on a Tietz $4 \mathrm{k} \times 4 \mathrm{k}$ CCD camera, TVIPS (Tietz Video and Image Processing Systems, GmbH, Gauting, Germany) at magnification of $\times 72,000$ (pixel size 2.08) and 1.3-2.5 $\mathrm{m}$ defocus. Micrographs for data collection were recorded on a DE-20 direct electron detector (Direct Electron, USA) at a magnification of $\times 83,000$ (pixel size $1.04 \AA$ ) and $0.8-2.1 \mu \mathrm{m}$ defocus. Images were recorded using a frame rate of 20 frames per second and $2 \mathrm{~s}$ exposure time (a total of 40 frames). The accumulated dose of the whole exposure was $\sim 62 \mathrm{e}^{-} \AA^{-2}$. A total of 24 images were recorded.

Processing of single particle images. For each exposure, the comprised frames were drift corrected using the DE_process_frames-2.7.1.py script ${ }^{64}$. Drift corrected images were imported to EMAN2 (version 2.12) for further processing ${ }^{65}$. Defocus, particle separation and amplitude contrast were evaluated with e2evalimage.py. Single particles, 3000, in different orientations were selected from the images using e2boxer.py in swarm or manual mode. False positives, such as aggregated particles and stain artifacts were discarded. For each image, the contrast transfer function (CTF) parameters were estimated on boxed out regions, $208 \times 208$ pixels, (containing particles) using the e2ctf.py program. A reference-free $2 \mathrm{D}$ classification was performed using 2,718 phase-flipped particles with e2refine2d.py. 2D classes, representing different orientations were selected for the initial 3D density map generation with e2initialmodel.py. These maps were low pass filtered to $60 \AA$. An approximate two-fold symmetry was revealed by $2 \mathrm{D}$-classification, in agreement with biochemical data. 3D refinement was performed using e2refine_easy.py applying both C2 and D2 symmetry aiming at a final resolution of $20 \AA$. Initial 3Drefinements were performed with pixel size of $4.152 \AA$ after binning the data by a linear factor 4 . In the second round of refinement, the data was binned to $2.076 \AA$ per pixel. The final map from the first round of refinements was used as input in the second. The resolution was determined based on a Fourier shell correlation (FSC) value of $0.143^{66}$, following the gold standard FSC procedure implemented in EMAN $2^{67}$. For testing the stability of $3 \mathrm{D}$-reconstructions a third round of refinement was performed with full sized data (1.038 $\AA$ per pixel).

Rh Bri2 BRICHOS species incubation in ThT buffer. To evaluate whether the different rh Bri2 BRICHOS species would be scrambled during the ThT assay observation period, rh Bri2 BRICHOS oligomers, dimers and monomers at concentrations $(10-15 \mu \mathrm{M})$, that are 2-3 times higher than the ones used in the ThT assay to enable the detection of small changes in assembly states, were incubated in ThT running buffer at $37^{\circ} \mathrm{C}$. Samples were taken out at different time points, i.e., 0 , 1,4 and $24 \mathrm{~h}$, and analyzed for assembly states by SDS-PAGE under reducing and non-reducing conditions.

A 342 monomer preparation and ThT assay. Recombinant $\mathrm{A} \beta$ (Met1-42), here referred to as $\mathrm{A} \beta 42$, was produced in BL2 $1^{\star}(\mathrm{DE} 3)$ pLysS E. coli (B strain) cells and purified by ion exchange ${ }^{18}$. Briefly, the IPTG-induced cells were lysed on ice by sonication for $3 \mathrm{~min}$ ( $2 \mathrm{~s}$ on and $2 \mathrm{~s}$ off, $65 \%$ maximum amplitude), and the pellets were collected by $24,000 \times g$ centrifugation at $4{ }^{\circ} \mathrm{C}$ for $10 \mathrm{~min}$. The pellets were dissolved by $8 \mathrm{M}$ urea in $10 \mathrm{mM}$ Tris- $\mathrm{HCl} \mathrm{pH} \mathrm{8.0,} \mathrm{which} \mathrm{was} \mathrm{then} \mathrm{diluted} \mathrm{with} 10$ $\mathrm{mM}$ Tris- $\mathrm{HCl} \mathrm{pH} 8.0$ to $2 \mathrm{M}$ urea for co-incubation (about $20 \mathrm{~min}$ in cold room) with DEAE cellulose (GE Healthcare, UK). The DEAE cellulose with bound proteins was washed with $10 \mathrm{mM}$ Tris- $\mathrm{HCl} \mathrm{pH} 8.0$ and $10 \mathrm{mM}$ Tris- $\mathrm{HCl} \mathrm{pH} 8.0$ containing $25 \mathrm{mM} \mathrm{NaCl}$, respectively, and the recombinant $\mathrm{A} \beta 42$ was finally eluted by $125 \mathrm{mM} \mathrm{NaCl}$ in $10 \mathrm{mM}$ Tris- $\mathrm{HCl} \mathrm{pH}$ 8.0. The eluate was passed through a 30 $\mathrm{kDa}$ concentration filter, and the filtrate (crude A $\beta 42$ ) was concentrated by a $5 \mathrm{kDa}$ concentration filter. The crude $A \beta 42$ proteins were lyophilized overnight and redissolved in $7 \mathrm{M} \mathrm{Gdn}-\mathrm{HCl}$ and then injected into a Superdex 75 column (GE Healthcare, UK) for monomer isolation in $20 \mathrm{mM}$ sodium phosphate $\mathrm{pH} 8.0$ with $0.2 \mathrm{mM}$ EDTA and $0.02 \% \mathrm{NaN} 3$. The A $\beta 42$ concentration was calculated by measuring the absorbance at 280 and $300 \mathrm{~nm}$ with an extinction coefficient of
$1,424 \mathrm{M}^{-1} \mathrm{~cm}^{-1}$ for $\left(\mathrm{A}_{280}-\mathrm{A}_{300}\right)$. Purified $\mathrm{A} \beta 42$ monomers were aliquoted in lowbinding Eppendorf tubes (Axygene). For analysis of the kinetics of amyloid fibril formation, $80 \mu \mathrm{L}$ solution containing $3 \mu \mathrm{M} \mathrm{A} \beta 42$ monomer, $10 \mu \mathrm{M}$ ThT and different concentrations of various rh Bri2 BRICHOS species were added to each well of half-area 96-well black polystyrene microplates with clear bottom and nonbinding surface (Corning Glass 3881, USA), and incubated under quiescent conditions at $37^{\circ} \mathrm{C}$. The aggregation kinetics of $\mathrm{A} \beta 42$ monomer with different concentrations in presence of a constant concentration $(0.9 \mu \mathrm{M})$ of various Bri2 BRICHOS species were measured in the same manner. The fluorescence was recorded using a $440 \mathrm{~nm}$ excitation filter and a $480 \mathrm{~nm}$ emission filter (FLUOStar Galaxy from BMG Labtech, Offenberg, Germany). For preparation of A $\beta 42$ seeds, $3 \mu \mathrm{M}$ A 342 monomer was incubated at $37^{\circ} \mathrm{C}$ for about $20 \mathrm{~h}$, and the fibrils were then sonicated in a water bath for $3 \mathrm{~min}$. For analysis of $\mathrm{A} \beta 42$ fibril formation kinetics in the presence of seeds, $80 \mu \mathrm{L}$ solution containing $3 \mu \mathrm{M} \mathrm{A} \beta 42$ monomer, $10 \mu \mathrm{M}$ ThT, different concentrations of rh Bri2 BRICHOS species, and $0.6 \mu \mathrm{M}$ seeds (calculated from the original A $\beta 42$ monomer concentration) were added at 4 ${ }^{\circ} \mathrm{C}$ to each well in triplicate of half-area 96-well plates and incubated under quiescent conditions at $37^{\circ} \mathrm{C}$. The fluorescence was recorded as described above. The initial slope of the concave aggregation traces was determined by a linear fit to the first $30 \mathrm{~min}$. Aggregation traces were normalized and averaged using 3-4 replicates for all the experiments. For A $\beta 42$ alone, averages were performed using two different runs with 3 replicates each.

Analysis of A $\mathbf{A 4 2}$ aggregation kinetics. Aggregation traces of $3 \mu \mathrm{M}$ A $\beta 42$ with different concentrations of Bri2 BRICHOS species (referring to the monomeric subunits) in molar ratios between 0 to $100 \%$ were, first, fitted to an empirical sigmoidal equation $^{39,40}$ :

$$
F=F_{0}+A /\left(1+\exp \left[r_{\max }\left(\tau_{1 / 2}-t\right)\right]\right)
$$

where $\tau_{1 / 2}$ is the aggregation half time, and $r_{\max }$ the maximal growth rate, $A$ the amplitude and $F_{0}$ the base value of the curve (Fig. $3 \mathrm{~d}$, e). The fitting parameter $\tau_{1 / 2}$ yields a linear and the $r_{\max }$ a mono-exponential decaying dependence on the relative Bri2 BRICHOS concentration.

Second, the aggregation kinetics was analyzed using a kinetic nucleation model that is determined by the microscopic rate constants for primary $\left(k_{n}\right)$ and secondary nucleation $\left(k_{2}\right)$ events as well as for fibril-end elongation $\left(k_{+}\right)^{17,37}$ Kinetic traces at different initial monomer concentration, $m(0)$, can be fitted globally, where the global fit parameters for primary $\lambda$ and secondary nucleation $\kappa$ are dependent on combined nucleation rates by $\lambda=\sqrt{2 \cdot k_{+} k_{n} \cdot m(0)^{n_{C}}}$ and $\kappa=\sqrt{2 \cdot k_{+} k_{2} \cdot m(0)^{n_{2}+1}}$. The parameters $n_{C}$ and $n_{2}$ are the reaction orders for primary and secondary nucleation, respectively. In this model the time dependence of the fibril mass $M(t)$ is given by ${ }^{17,38}$ :

$$
\frac{M(t)}{M(\infty)}=1-\left(\frac{B_{+}+C_{+}}{B_{+}+C_{+} \cdot \exp (\kappa t)} \cdot \frac{B_{-}+C_{+} \cdot \exp (\kappa t)}{B_{-}+C_{+}}\right)^{\frac{k_{\infty}^{2}}{\kappa k_{\infty}}} \cdot \exp \left(-k_{\infty} t\right)
$$

where the additional coefficients are functions of $\lambda$ and $\kappa$ :

$$
\begin{aligned}
& C_{ \pm}= \pm \lambda^{2} / 2 / \kappa^{2} \\
& k_{\infty}=\sqrt{2 \kappa^{2} /\left(n_{2}\left(n_{2}+1\right)\right)+2 \lambda^{2} / n_{C}} \\
& \tilde{k}_{\infty}=\sqrt{k_{\infty}^{2}-4 C_{+} C_{-} \kappa^{2}} \\
& B_{ \pm}=\left(k_{\infty} \pm \tilde{k}_{\infty}\right) / 2 / \kappa
\end{aligned}
$$

The kinetic data at constant A $\beta 42$ concentration with different Bri2 BRICHOS concentrations were then analyzed again applying the kinetic nucleation model First, each kinetic trace was fitted individually with the kinetic nucleation model described by Eq. 1 (Fig. 3a-c).

Aggregation traces of $A \beta 42$ alone exhibit a $\gamma$-exponent of $\gamma=-1.56 \pm 0.10$ (Fig. 4a), which corresponds to a model including monomer-dependent secondary nucleation in addition to fibril-end elongation, described by Eq. 1. The aggregation traces were, second, fitted globally (Supplementary Fig. 5) and the model fits well the data when the reaction orders for primary and secondary nucleation are set to $n_{C}=n_{2}=2$, as reported previously for $A \beta 42$ aggregation ${ }^{17}$. The results from the global fit are given in Supplementary Table 2.

Also, the aggregation traces in the presence of Bri2 BRICHOS could be fitted globally with the same model as for A $\beta 42$ alone (Supplementary Fig. 5) and the global fitting parameters are listed in Supplementary Table 2. The fitting parameter for primary nucleation, $\lambda$, could be fixed to the same value as for A $\beta 42$ alone for all Bri2 BRICHOS species.

We also performed a global fit of the kinetic data set at constant A $\beta 42$ concentration and different Bri2 BRICHOS concentration where the fit was constrained such that one fitting parameter was hold to a constant value across all Bri2 BRICHOS concentration, while the second parameter was the only free parameter (Supplementary Fig. 7 and Supplementary Table 3). This fitting constraint results in that only one single rate constant, i.e., $k_{n}, k_{+}$or $k_{2}$, is the sole fitting parameter ${ }^{39,40}$. 
Immunogold staining of A $\mathbf{\beta 4 2}$ fibrils. In total $5 \mu \mathrm{M}$ A $\beta 42$ monomer was incubated at $37^{\circ} \mathrm{C}$ with rh Bri2 BRICHOS monomer and dimer, respectively, for about $15 \mathrm{~h}$, and the fibrils were collected at $4^{\circ} \mathrm{C}$ by centrifugation for $1 \mathrm{~h}$ at $22,000 \times g$. The fibrils were carefully resuspended in $20 \mu \mathrm{L}$ TBS, of which $2 \mu \mathrm{L}$ were applied to formvar coated nickel grids, and incubated for about $5 \mathrm{~min}$. Excess solution was removed with the edge of a Kleenex paper towel. Blocking was performed by incubation in $1 \%$ BSA in TBS for $30 \mathrm{~min}$, after which the grids were washed $3 \times 10$ min by TBS. The grids were then incubated with goat anti-Bri2 BRICHOS antibody (1:200 dilution) overnight at $4^{\circ} \mathrm{C}$, and washed $3 \times 10 \mathrm{~min}$ with TBS. Finally the grids were incubated with anti-goat IgG-gold secondary antibody (1:40 dilution) coupled to $10 \mathrm{~nm}$ gold particles (BBI Solutions, UK, EM.RAG10) for $2 \mathrm{~h}$ at room temperature, and washed $5 \times 10 \mathrm{~min}$ with TBS. Excess solution was removed from the grid surface with the edge of a Kleenex paper towel. For staining, $2 \mu \mathrm{L}$ of $2.5 \%$ uranyl acetate was added on each grid (kept about $20 \mathrm{~s}$ ), and excess solution was finally removed. The grids were dried for about $20 \mathrm{~s}$, and analyzed by transmission electron microscopy (TEM, Hitachi H7100 TEM operated at $75 \mathrm{kV}$ ).

Electrophysiological studies with rh Bri2 BRICHOS species. Experiments were carried out in accordance with the ethical permit granted by Norra Stockholm's Djurförsöksetiska Nämnd (dnr N45/13 to AF). C57BL/6 mice of either sex (postnatal days 14-23, supplied from Charles River, Germany) were used in the experiments. The animals were deeply anaesthetized using isofluorane before being sacrificed by decapitation.

The brain was dissected out and placed in ice-cold ACSF (artificial cerebrospinal fluid) modified for dissection. This solution contained $80 \mathrm{mM} \mathrm{NaCl}$, $24 \mathrm{mM} \mathrm{NaHCO} 3,25 \mathrm{mM}$ glucose, $1.25 \mathrm{mM} \mathrm{NaH}_{2} \mathrm{PO}_{4}, 1 \mathrm{mM}$ ascorbic acid, $3 \mathrm{mM}$ NaPyruvate, $2.5 \mathrm{mM} \mathrm{KCl}, 4 \mathrm{mM} \mathrm{MgCl}_{2}, 0.5 \mathrm{mM} \mathrm{CaCl}_{2}$ and $75 \mathrm{mM}$ sucrose. Horizontal sections ( $350 \mu \mathrm{m}$ thick) of the ventral hippocampi of both hemispheres were prepared with a Leica VT1200S vibratome (Microsystems, Stockholm, Sweden) and treated under different experimental conditions (see below) without randomization and the conditions were not blinded to the experimenter. Immediately after slicing sections were transferred to a submerged incubation chamber containing standard ACSF: $124 \mathrm{mM} \mathrm{NaCl}, 30 \mathrm{mM} \mathrm{NaHCO}, 10 \mathrm{mM}$ glucose, $1.25 \mathrm{mM} \mathrm{NaH}_{2} \mathrm{PO}_{4}, 3.5 \mathrm{mM} \mathrm{KCl}, 1.5 \mathrm{mM} \mathrm{MgCl}_{2}$ and $1.5 \mathrm{mM} \mathrm{CaCl}_{2}$. The chamber was held at $34^{\circ} \mathrm{C}$ for at least $20 \mathrm{~min}$ after dissection. It was subsequently allowed to cool to ambient room temperature $\left(\sim 22^{\circ} \mathrm{C}\right)$ for a minimum of $40 \mathrm{~min}$. Proteins (A $\beta 42$, Bri2 BRICHOS species and combinations thereof) were added to the incubation solution for $15 \mathrm{~min}$ before transferring slices to the interface-style recording chamber for extracellular recordings. While incubating, slices were continuously supplied with carbogen gas $\left(5 \% \mathrm{CO}_{2}, 95 \% \mathrm{O}_{2}\right)$ bubbled into the ACSF.

Recordings were carried out in hippocampal area CA3 with borosilicate glass microelectrodes, pulled to a resistance of 3-5 M $\Omega$. Local field potentials (LFP) were recorded in an interface-type chamber (perfusion rate $4.5 \mathrm{~mL} \mathrm{~min}^{-1}$ ) at $32{ }^{\circ} \mathrm{C}$ using microelectrodes filled with ACSF placed in stratum pyramidale. LFP $\gamma$ oscillations was elicited by applying kainic acid (KA) $(100 \mathrm{nM}$, Tocris) to the extracellular bath. The oscillations were allowed to stabilize for $20 \mathrm{~min}$ before any recordings were carried out. No A $\beta 42$, rh Bri2 BRICHOS species and combinations thereof were present in the recording chamber either during stabilization of $\gamma$ oscillations or thereafter during electrophysiological recordings. The interface chamber recording solution contained $124 \mathrm{mM} \mathrm{NaCl}, 30 \mathrm{mM} \mathrm{NaHCO}, 10 \mathrm{mM}$ glucose, $1.25 \mathrm{mM}$ $\mathrm{NaH}_{2} \mathrm{PO}_{4}, 3.5 \mathrm{mM} \mathrm{KCl}, 1.5 \mathrm{mM} \mathrm{MgCl}$, and $1.5 \mathrm{mM} \mathrm{CaCl}_{2}$

Interface chamber LFP recordings were performed with a 4-channel amplifier/ signal conditioner M102 amplifier (Electronics lab, Faculty of Mathematics and Natural Sciences, University of Cologne, Cologne, Germany). The signals were sampled at $10 \mathrm{kHz}$, conditioned using a Hum Bug $50 \mathrm{~Hz}$ noise eliminator (LFP signals only; Quest Scientific, North Vancouver, BC, Canada), software low-pass filtered at $1 \mathrm{kHz}$, digitized and stored using a Digidata $1322 \mathrm{~A}$ and Clampex 9.6 software (Molecular Devices, CA, USA).

Power spectral density plots (from $60 \mathrm{~s}$ long LFP recordings) were calculated in averaged Fourier-segments of 8,192 points using Axograph X (Kagi, Berkeley, CA, USA). Oscillation power was calculated by integrating the power spectral density between 20 and $80 \mathrm{~Hz}$. Data is reported as means \pm standard errors of the means. For statistical analysis the Student's $t$-test (unpaired) was used. Significance levels are ${ }^{*} p<0.05 ;{ }^{* *} p<0.01 ;{ }^{* *} p<0.001$. All experiments were performed with parallel controls from the same animal/preparation.

Bri2 BRICHOS incubation in buffer and in serum. The procedures performed involving animal tissues were in accordance with the ethical permit granted from Stockholm Södra Djurförsöksetiska Nämnd (dnr S 6-15). Adult male mice C57BL/ 6 were anesthetized with a solution of ketamine/xylazine, then the thoracic cavity was carefully opened and the heart exposed. Intracardial blood collection was performed with a $0.5 \mathrm{~mL}$ syringe from the right atrium. From each mouse 0.5 to 1 $\mathrm{mL}$ blood was collected, coagulated and centrifuged at $10,000 \mathrm{rpm}$ for $15 \mathrm{~min}$ at $4^{\circ}$ $\mathrm{C}$ to collect serum. Rh Bri2 BRICHOS monomer $(5 \mu \mathrm{M})$ was incubated at $37^{\circ} \mathrm{C}$ in $80 \%(\mathrm{v} / \mathrm{v})$ serum overnight, and serum alone with same amount of PBS as control. Samples were taken out at different time points, diluted in PBS, and added to reducing or non-reducing SDS loading buffer. The samples were separated using $13.5 \%$ SDS-PAGE gels and the oligomerization was analyzed by western blotting with polyclonal goat anti-Bri2 BRICHOS antibody. Additionally, monomeric rh
Bri2 BRICHOS $(26.8 \mu \mathrm{M})$ in buffer of $20 \mathrm{mM}$ phosphate $\mathrm{pH} 8.0$ containing $0.2 \mathrm{mM}$ EDTA and $0.02 \% \mathrm{NaN}_{3}$ was incubated at $37^{\circ} \mathrm{C}$ overnight, and the degree of oligomerization was analyzed by SDS-PAGE under reducing and non-reducing conditions, the activity against A $\beta 42$ fibrillization was evaluated using ThT assay described above and the activity against non-fibrillar aggregation was measured as described below. As a control, $20 \mu \mathrm{M}$ rh proSP-C BRICHOS (residues 86-197 according to the full-length sequence) was incubated in $20 \mathrm{mM}$ phosphate $\mathrm{pH} 8.0$ containing $0.2 \mathrm{mM}$ EDTA and $0.02 \% \mathrm{NaN} 3$ at $37^{\circ} \mathrm{C}$ overnight. The oligomerization and activity against $\mathrm{A} \beta 42$ fibrillization was analyzed in the same manner as for rh Bri2 BRICHOS.

CS non-fibrillar aggregation. CS from porcine heart (Sigma-Aldrich, Germany) in $40 \mathrm{mM}$ HEPES/KOH pH 7.5 was diluted to $600 \mathrm{nM}$ in the same buffer and then equilibrated at $45^{\circ} \mathrm{C}$ in the presence or absence of different concentrations of various rh Bri2 BRICHOS species. The aggregation kinetics were measured in triplicate using a microplate reader (FLUOStar Galaxy from BMG Labtech, Offenberg, Germany) by reading the apparent increase in absorbance at $360 \mathrm{~nm}$ during incubation at $45^{\circ} \mathrm{C}$ under quiescent conditions. The activity against CS aggregation of rh Bri2 BRICHOS monomers incubated at $37^{\circ} \mathrm{C}$ was analyzed, using an UV-Visible Spectrophotometer (300 Bio, CARY) with thermostated quartz cuvette, by reading the apparent increase in absorbance at $360 \mathrm{~nm}$.

For analysis of CS aggregation in the presence of serum-incubated rh Bri2 BRICHOS monomers, the incubation aliquots were collected at $0 \mathrm{~h}$ and after $24 \mathrm{~h}$. CS was diluted to $600 \mathrm{nM}$ and mixed with $600 \mathrm{nM}$ serum-incubated rh Bri2 BRICHOS monomers or corresponding volume of PBS, and analyzed in triplicate. Additional samples containing equal volumes of serum incubated without Bri2 BRICHOS for 0 or $24 \mathrm{~h}$ were analyzed. Five replicates per sample were pipetted in half-area 96-well black polystyrene microplates with clear bottom and nonbinding surface (Corning Glass 3881, USA), each containing $150 \mu \mathrm{L}$ samples. The aggregation was measured in a microplate reader as described above. The data were baseline corrected for each individual measurement, averaging the replicates and subtracting the corresponding blank from each CS containing sample.

Rh Bri2 BRICHOS from mammalian cells and deglycosylation. Human embryonic kidney (HEK) cell derived HEK293 cells (Thermo Fisher Scientific, USA) were seeded and cultured with Expi293TM Expression Media until about 200 million viable cells were obtained. The cells were not authenticated or regularly tested for mycoplasma infection. Cells were transfected with $250 \mu \mathrm{g}$ plasmid DNA, coding for human Bri2 BRICHOS (residues 113-231) tagged C-terminally with the antibody epitope AU1, using $1 \mathrm{mg} \mathrm{mL}^{-1}$ PEI (Sigma Aldrich, Germany) and cultured for 12 days. The recombinant proteins were directed to the secretory pathway with the prosurfactant protein B signal peptide (residues $1-23)^{68}$. The conditioned media or cell lysates were loaded into a HisTrap excel $1 \times 1 \mathrm{~mL}$ columns (Thermo Fisher Scientific, USA), then eluted with $20 \mathrm{mM}$ sodium phosphate $\mathrm{pH} 7.4$ containing $300 \mathrm{mM}$ imidazole and analyzed by western blotting with primary polyclonal rabbit anti-AU1 antibody (1:1000 dilution, Abcam, UK, ab3401) and secondary polyclonal donkey anti-rabbit IgG/HRP antibody (1:5000 dilution, GE Healthcare, UK, NA934). Alternatively, HEK293 cells $\left(7 \times 10^{5}\right.$ cells per flask) were seeded into T75 cell culture flasks and cultured with Dulbecco's modified Eagels's medium (Gibco ${ }^{\mathrm{TM}}$ DMEM, high glucose, GlutaMAX ${ }^{\mathrm{TM}}$; Thermo Fisher Scientific USA) containing 10\% FBS (Gibco ${ }^{\mathrm{TM}}$ Thermo Fisher Scientific, USA) until $90 \%$ confluence. Cells were transfected with $1.0 \mu \mathrm{g}$ plasmid DNA, encoding rh Bri2 BRICHOS-AU1 in a pcDNA3.4-TOPO vector using $0.03 \mathrm{mg} \mathrm{mL}^{-1}$ Lipofectamine $^{\circledR}$ 2000 (Thermo Fisher Scientific, Waltham, USA). As control no plasmid was transfected. During protein expression cells were cultured in FBS free DMEM medium for $24 \mathrm{~h}$. The transfected cells were incubated with $\mathrm{N}$-Ethylmaleimid (NEM, Sigma-Aldrich, Germany) diluted in PBS pH 7.4 to a final concentration of $50 \mathrm{mM}$ for $10 \mathrm{~min}$ at room temperature; equal amounts of PBS were used as a control. The cells were lysed in lysis buffer (100 mM Tris- $\mathrm{HCl}, 200 \mathrm{mM} \mathrm{NaCl}, 2$ mM EDTA, 2\% Triton-X), containing $50 \mathrm{mM}$ NEM. The total protein concentration was determined using the Bradford protein assay (Bio-Rad, Germany) Presence of N-linked glycans of rh Bri2 BRICHOS was analyzed by incubating control and transfected HEK293 cell lysates with 5 U N-Glycosidase F (PNGase F) (Roche, Mannheim, Germany) overnight at $37^{\circ} \mathrm{C}$. Reduced and non-reduced samples ( $50 \mu \mathrm{g}$ total protein/sample) were prepared and loaded on $13.5 \%$ SDS gels, and subsequently analyzed by western blotting with primary polyclonal rabbit antiAU1 antibody (1:1000 dilution, Abcam, UK, ab3401) and secondary polyclonal donkey anti-rabbit IgG/HRP antibody (1:5000 dilution, GE Healthcare, UK, NA934), and visualized in a CCD camera (Fujifilm LAS-3000, Japan).

Cells of the human neuroblastoma derived cell line SH-SY5Y $\left(7 \times 10^{5}\right.$ cells per flask) (ATCC, Sweden) were seeded into T25 flasks and cultured in DMEM/F-12 medium (Gibco ${ }^{\mathrm{TM}}$ DMEM/F-12, GlutaMAX ${ }^{\mathrm{TM}}$; Thermo Fisher Scientific, USA), containing $10 \%$ FBS until $60 \%$ confluence was reach. The cells were not authenticated or regularly tested for mycoplasma infection. The cell culture was continued for $24 \mathrm{~h}$ with FBS free medium. Before the medium was collected NEM or PBS as a control, was added as described above. Prior to immunoprecipitation detached cells were removed by centrifugation from the media and pre-cleared with Protein A sepharose (GE Healthcare, UK) for $1 \mathrm{~h}$ at $4{ }^{\circ} \mathrm{C}$. The supernatants were incubated with polyclonal goat anti-Bri2 113-231 antibody (1:250 dilution) for $1 \mathrm{~h}$ at $4{ }^{\circ} \mathrm{C}$ and samples were incubated with Protein A sepharose for $1 \mathrm{~h}$ at $4{ }^{\circ} \mathrm{C}$ 
After washing the sepharose three times with PBS, SDS buffer was added and the samples were heated at $96^{\circ} \mathrm{C}$ for $10 \mathrm{~min}$. The analysis was done by western blotting with using primary polyclonal goat anti-Bri2 113-231 antibody (1:250 dilution) and secondary polyclonal rabbit anti-goat IgG/HRP antibody (1:5000 dilution, Thermo Fisher Scientific, USA, 61-1620), and visualized using a CCD camera (Fujifilm LAS-3000, Japan).

Data availability. The density map of the Bri2 BRICHOS oligomer have been deposited in the Electron Microscopy Data Bank (EMDB) under the accession code EMD-3918. The other data that support the findings of this study are available from the corresponding author upon reasonable request.

Received: 6 July 2017 Accepted: 2 November 2017

Published online: 12 December 2017

\section{References}

1. Balchin, D., Hayer-Hartl, M. \& Hartl, F. U. In vivo aspects of protein folding and quality control. Science 353, aac4354 (2016).

2. Bucciantini, M. et al. Inherent toxicity of aggregates implies a common mechanism for protein misfolding diseases. Nature 416, 507-511 (2002).

3. Balch, W. E., Morimoto, R. I., Dillin, A. \& Kelly, J. W. Adapting proteostasis for disease intervention. Science 319, 916-919 (2008).

4. Chiti, F. \& Dobson, C. M. Protein misfolding, amyloid formation, and human disease: a summary of progress over the last decade. Annu. Rev. Biochem. 86, 27-68 (2017).

5. Sipe, J. D. et al. Amyloid fibril proteins and amyloidosis: chemical identification and clinical classification International Society of Amyloidosis 2016 Nomenclature Guidelines. Amyloid 23, 209-213 (2016).

6. Carra, S. et al. The growing world of small heat shock proteins: from structure to functions. Cell Stress Chaperones 22, 601-611 (2017).

7. Hayer-Hartl, M., Bracher, A. \& Hartl, F. U. The GroEL-GroES chaperonin machine: a nano-cage for protein folding. Trends Biochem. Sci. 41, 62-76 (2016).

8. Mashaghi, A. et al. Alternative modes of client binding enable functional plasticity of Hsp70. Nature 539, 448-451 (2016).

9. Goldschmidt, L., Teng, P. K., Riek, R. \& Eisenberg, D. Identifying the amylome, proteins capable of forming amyloid-like fibrils. Proc. Natl Acad. Sci. USA 107, 3487-3492 (2010)

10. Evans, C. G., Wisen, S. \& Gestwicki, J. E. Heat shock proteins 70 and 90 inhibit early stages of amyloid beta-(1-42) aggregation in vitro. J. Biol. Chem. 281, 33182-33191 (2006)

11. Arimon, M., Grimminger, V., Sanz, F. \& Lashuel, H. A. Hsp104 targets multiple intermediates on the amyloid pathway and suppresses the seeding capacity of Abeta fibrils and protofibrils. J Mol. Biol. 384, 1157-1173 (2008).

12. Warrick, J. M. et al. Suppression of polyglutamine-mediated neurodegeneration in Drosophila by the molecular chaperone HSP70. Nat. Genet. 23, 425-428 (1999).

13. Kakkar, V. et al. The S/T-Rich motif in the DNAJB6 chaperone delays polyglutamine aggregation and the onset of disease in a mouse model. Mol. Cell https://doi:10.1016/j.molcel.2016.03.017 (2016).

14. Hardy, J. The discovery of Alzheimer-causing mutations in the APP gene and the formulation of the "amyloid cascade hypothesis". FEBS J. 284, 1040-1044 (2017).

15. Walsh, D. M. et al. Naturally secreted oligomers of amyloid beta protein potently inhibit hippocampal long-term potentiation in vivo. Nature 416, 535-539 (2002).

16. Haass, C. \& Selkoe, D. J. Soluble protein oligomers in neurodegeneration: lessons from the Alzheimer's amyloid beta-peptide. Nat. Rev. Mol Cell Biol. 8, 101-112 (2007)

17. Cohen, S. I. et al. Proliferation of amyloid-beta42 aggregates occurs through a secondary nucleation mechanism. Proc. Natl Acad. Sci. USA 110, 9758-9763 (2013).

18. Cohen, S. I. et al. A molecular chaperone breaks the catalytic cycle that generates toxic Abeta oligomers. Nat. Struct. Mol. Biol. 22, 207-213 (2015).

19. Hedlund, J., Johansson, J. \& Persson, B. BRICHOS-a superfamily of multidomain proteins with diverse functions. BMC Res Notes 2, 180 (2009).

20. Sanchez-Pulido, L., Devos, D. \& Valencia, A. BRICHOS: a conserved domain in proteins associated with dementia, respiratory distress and cancer. Trends Biochem. Sci. 27, 329-332 (2002).

21. Akiyama, H. et al. Expression of BRI, the normal precursor of the amyloid protein of familial British dementia, in human brain. Acta Neuropathol. 107, 53-58 (2004).

22. Vidal, R. et al. A stop-codon mutation in the BRI gene associated with familial British dementia. Nature 399, 776-781 (1999).
23. Matsuda, S. et al. The familial dementia BRI2 gene binds the Alzheimer gene amyloid-beta precursor protein and inhibits amyloid-beta production. J. Biol. Chem. 280, 28912-28916 (2005).

24. Del Campo, M. et al. BRI2-BRICHOS is increased in human amyloid plaques in early stages of Alzheimer's disease. Neurobiol. Aging 35, 1596-1604 (2014).

25. Vidal, R. et al. A decamer duplication in the 3 ' region of the BRI gene originates an amyloid peptide that is associated with dementia in a Danish kindred. Proc Natl Acad. Sci. USA 97, 4920-4925 (2000).

26. Willander, $\mathrm{H}$. et al. BRICHOS domains efficiently delay fibrillation of amyloid beta-peptide. J. Biol. Chem. 287, 31608-31617 (2012).

27. Buxbaum, J. N. \& Johansson, J. Transthyretin and BRICHOS: the paradox of amyloidogenic proteins with anti-amyloidogenic activity for abeta in the central nervous system. Front. Neurosci. 11, 119 (2017).

28. Poska, H. et al. Dementia-related Bri2 BRICHOS is a versatile molecular chaperone that efficiently inhibits Abeta 42 toxicity in Drosophila. Biochem. J. 473, 3683-3704 (2016)

29. Arosio, P. et al. Kinetic analysis reveals the diversity of microscopic mechanisms through which molecular chaperones suppress amyloid formation. Nat. Commun. 7, 10948 (2016).

30. Singh, J. K. \& Balch, W. E. Proteostatic hotspots in amyloid fibrils protect us from neurodegeneration. Dev. Cell 32, 659-660 (2015).

31. Kronqvist, N. et al. Efficient protein production inspired by how spiders make silk. Nat. Commun. 8, 15504 (2017).

32. Willander, H. et al. High-resolution structure of a BRICHOS domain and its implications for anti-amyloid chaperone activity on lung surfactant protein $\mathrm{C}$ Proc. Natl Acad. Sci. USA 109, 2325-2329 (2012).

33. Hoffmann, A., Bukau, B. \& Kramer, G. Structure and function of the molecular chaperone Trigger Factor. Biochim. Biophys. Acta 1803, 650-661 (2010).

34. Hawe, A., Sutter, M. \& Jiskoot, W. Extrinsic fluorescent dyes as tools for protein characterization. Pharm. Res. 25, 1487-1499 (2008).

35. Cohen, S. I. A., Vendruscolo, M., Dobson, C. M. \& Knowles, T. P. J. From macroscopic measurements to microscopic mechanisms of protein aggregation. J. Mol. Biol. 421, 160-171 (2012).

36. Knowles, T. P. J. et al. An analytical solution to the kinetics of breakable filament assembly. Science 326, 1533-1537 (2009)

37. Cohen, S. I. A. et al. Nucleated polymerization with secondary pathways. I. Time evolution of the principal moments. J. Chem. Phys. 135, 065105 (2011).

38. Cohen, S. I. A., Vendruscolo, M., Dobson, C. M. \& Knowles, T. P. J. Nucleated polymerization with secondary pathways. II. Determination of self-consistent solutions to growth processes described by non-linear master equations. J. Chem. Phys. 135, 065106 (2011).

39. Abelein, A., Gräslund, A. \& Danielsson, J. Zinc as chaperone-mimicking agent for retardation of amyloid $\beta$ peptide fibril formation. Proc. Natl Acad. Sci. USA 112, 5407-5412 (2015).

40. Abelein, A., Jarvet, J., Barth, A., Gräslund, A. \& Danielsson, J. Ionic strength modulation of the free energy landscape of A $\beta 40$ peptide fibril formation. $J$. Am. Chem. Soc. 138, 6893-6902 (2016).

41. Buzsaki, G. Rhythms of the brain (Oxford University Press, Oxford 2006).

42. Yamamoto, J., Suh, J., Takeuchi, D. \& Tonegawa, S. Successful execution of working memory linked to synchronized high-frequency gamma oscillations. Cell 157, 845-857 (2014).

43. Ribary, U. et al. Magnetic field tomography of coherent thalamocortical $40-\mathrm{Hz}$ oscillations in humans. Proc. Natl Acad. Sci. USA 88, 11037-11041 (1991).

44. Garringer, H. J., Sammeta, N., Oblak, A., Ghetti, B. \& Vidal, R. Amyloid and intracellular accumulation of BRI2. Neurobiol. Aging 52, 90-97 (2017).

45. Ogura, T. et al. Three-dimensional structure of the gamma-secretase complex. Biochem. Biophys. Res. Commun. 343, 525-534 (2006).

46. Biverstal, H. et al. Dissociation of a BRICHOS trimer into monomers leads to increased inhibitory effect on Abeta42 fibril formation. Biochim. Biophys. Acto 1854, 835-843 (2015).

47. Delbecq, S. P. \& Klevit, R. E. One size does not fit all: the oligomeric states of alphaB crystallin. FEBS Lett. 587, 1073-1080 (2013).

48. Basha, E., O’Neill, H. \& Vierling, E. Small heat shock proteins and alphacrystallins: dynamic proteins with flexible functions. Trends Biochem. Sci. 37, 106-117 (2012).

49. Rutsdottir, G. et al. Structural model of dodecameric heat-shock protein Hsp21 flexible $\mathrm{N}$-terminal arms interact with client proteins while C-terminal tails maintain the dodecamer and chaperone activity. J. Biol. Chem. 292, 8103-8121 (2017)

50. Haslbeck, M. \& Vierling, E. A first line of stress defense: small heat shock proteins and their function in protein homeostasis. J. Mol. Biol. 427, 1537-1548 (2015).

51. Benesch, J. L., Ayoub, M., Robinson, C. V. \& Aquilina, J. A. Small heat shock protein activity is regulated by variable oligomeric substructure. J. Biol. Chem. 283, 28513-28517 (2008)

52. Wyatt, A. R., Yerbury, J. J., Ecroyd, H. \& Wilson, M. R. Extracellular chaperones and proteostasis. Annu. Rev. Biochem. 82, 295-322 (2013). 
53. Shammas, S. L. et al. Binding of the molecular chaperone alphaB-crystallin to Abeta amyloid fibrils inhibits fibril elongation. Biophys. J. 101, 1681-1689 (2011).

54. Månsson, C. et al. Interaction of the molecular chaperone DNAJB6 with growing amyloid-beta 42 (Abeta42) aggregates leads to sub-stoichiometric inhibition of amyloid formation. J. Biol. Chem. 289, 31066-31076 (2014).

55. Mainz, A. et al. The chaperone alphaB-crystallin uses different interfaces to capture an amorphous and an amyloid client. Nat. Struct. Mol. Biol. 22, 898-905 (2015).

56. Wilson, M. R. \& Zoubeidi, A. Clusterin as a therapeutic target. Exp. Opin. Ther. Targets 21, 201-213 (2017).

57. Bonito-Oliva, A., Barbash, S., Sakmar, T. P. \& Graham, W. V. Nucleobindin 1 binds to multiple types of pre-fibrillar amyloid and inhibits fibrillization. Sci. Rep. 7, 42880 (2017).

58. Yerbury, J. J. et al. The extracellular chaperone clusterin influences amyloid formation and toxicity by interacting with prefibrillar structures. FASEB J. 21, 2312-2322 (2007).

59. Poon, S. et al. Mildly acidic $\mathrm{pH}$ activates the extracellular molecular chaperone clusterin. J. Biol. Chem. 277, 39532-39540 (2002).

60. Colvin, M. T. et al. Atomic resolution structure of monomorphic Abeta42 amyloid fibrils. J. Am. Chem. Soc. 138, 9663-9674 (2016).

61. Wälti, M. A. et al. Atomic-resolution structure of a disease-relevant Abeta(1-42) amyloid fibril. Proc. Natl Acad. Sci. USA 113, E4976-E4984 (2016).

62. Martin, L. et al. Regulated intramembrane proteolysis of Bri2 (Itm2b) by ADAM10 and SPPL2a/SPPL2b. J. Biol. Chem. 283, 1644-1652 (2008).

63. Tsachaki, M., Ghiso, J., Rostagno, A. \& Efthimiopoulos, S. BRI2 homodimerizes with the involvement of intermolecular disulfide bonds. Neurobiol. Aging 31, 88-98 (2010).

64. Bammes, B. E., Chen, D. H., Jin, L. \& Bilhorn, R. B. Visualizing and correcting dynamic specimen processes in TEM using a direct detection device. Microscopy Microanal. 19, 1320-1321 (2013).

65. Tang, G. et al. EMAN2: an extensible image processing suite for electron microscopy. J. Struct. Biol. 157, 38-46 (2007).

66. Rosenthal, P. B. \& Henderson, R. Optimal determination of particle orientation, absolute hand, and contrast loss in single-particle electron cryomicroscopy. J. Mol. Biol. 333, 721-745 (2003).

67. Scheres, S. H. \& Chen, S. Prevention of overfitting in cryo-EM structure determination. Nat. Methods 9, 853-854 (2012).

68. Dolfe, L., Winblad, B., Johansson, J. \& Presto, J. BRICHOS binds to a designed amyloid-forming beta-protein and reduces proteasomal inhibition and aggresome formation. Biochem. J. 473, 167-178 (2016).

\section{Acknowledgements}

We are grateful to Drs. Claes Andréasson and Anna Rising for helpful comments on this manuscript. This study was supported by a KID PhD studentship grant (FRK), the
Swedish Research Council (J.J., A.F., H.H.), the Center for Innovative Medicine (CIMED) (J.J., H.H.), the Swedish Alzheimer foundation (A.F.), the Swedish Brain Foundation (A.F.), the Strategic Program in Neurosciences at the Karolinska Institutet (A.F.), the Stiftelsen för Gamla Tjänarinnor (G.C., A.A., H.B.), Instruct R\&D pilot project grant APPID 272 (A.A.), Loo and Hans Osterman Foundation (G.C., A.A.), Geriatric Diseases Foundation at Karolinska Institutet (G.C., A.F.), VIAA Latvia NFI/R/ 2014/023 grant (H.B.) and the InnovaBalt project at Latvian Institute of Organic Synthesis (H.B.).

\section{Author contributions}

G.C., H.E.N. A.L., Y.A.T., S.T., L.H., F.R., M.L. performed experiments. A.A. performed the kinetic analyses. G.C., A.A, H.E.N., H.H., P.J.B.K., J.P., H.B., A.F., and J.J. analyzed the data. J.J. conceived and supervised the study. G.C., A.A., and J.J. wrote the paper. All authors discussed the results and commented on the manuscript.

\section{Additional information}

Supplementary Information accompanies this paper at https://doi.org/10.1038/s41467017-02056-4.

Competing interests: The authors declare no competing financial interests.

Reprints and permission information is available online at http://npg.nature.com/ reprintsandpermissions/

Publisher's note: Springer Nature remains neutral with regard to jurisdictional claims in published maps and institutional affiliations.

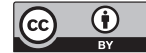

Open Access This article is licensed under a Creative Commons Attribution 4.0 International License, which permits use, sharing, adaptation, distribution and reproduction in any medium or format, as long as you give appropriate credit to the original author(s) and the source, provide a link to the Creative Commons license, and indicate if changes were made. The images or other third party material in this article are included in the article's Creative Commonslicense, unless indicated otherwise in a credit line to the material. If material is not included in the article'sCreative Commons license and your intended use is not permitted by statutory regulation or exceeds the permitted use, you will need to obtain permission directly from the copyright holder. To view a copy of this license, visit http://creativecommons.org/ licenses/by/4.0/.

(C) The Author(s) 2017 